\title{
Orion Crew Exploration Vehicle Launch Abort System Guidance and Control Analysis Overview
}

\author{
John B. Davidson ${ }^{1}$, Sungwan Kim² ${ }^{2}$ David L. Raney ${ }^{3}$, \\ Vanessa V. Aubuchon ${ }^{4}$, Dean W. Sparks ${ }^{5}$, and Ronald C. Busan ${ }^{6}$ \\ NASA Langley Research Center, Hampton, VA 23681 \\ Ryan W. Proud ${ }^{7}$ and Deborah S. Merritt ${ }^{8}$ \\ NASA Johnson Space Center, Houston, TX 77058
}

\begin{abstract}
Aborts during the critical ascent flight phase require the design and operation of Orion Crew Exploration Vehicle (CEV) systems to escape from the Crew Launch Vehicle (CLV) and return the crew safely to the Earth. To accomplish this requirement of continuous abort coverage, CEV ascent abort modes are being designed and analyzed to accommodate the velocity, altitude, atmospheric, and vehicle configuration changes that occur during ascent. Aborts from the launch pad to early in the flight of the CLV second stage are performed using the Launch Abort System (LAS). During this type of abort, the LAS Abort Motor is used to pull the Crew Module (CM) safely away from the CLV and Service Module (SM). LAS abort guidance and control studies and design trades are being conducted so that more informed decisions can be made regarding the vehicle abort requirements, design, and operation. This paper presents an overview of the Orion CEV, an overview of the LAS ascent abort mode, and a summary of key LAS abort analysis methods and results.
\end{abstract}

\section{Nomenclature}

Symbols

$\alpha=$ angle of attack

$\mathrm{A}_{\text {ref }} \quad=$ vehicle reference area

$\mathrm{C}_{\mathrm{m}} \quad=$ pitching moment coefficient

$\mathrm{C}_{\mathrm{m}_{\alpha}} \quad=$ static longitudinal stability $\partial \mathrm{C}_{\mathrm{m}} / \partial \alpha$

$\mathrm{h}=$ altitude

$1_{\text {ref }} \quad=$ vehicle reference length

$\mathrm{M} \quad=$ Mach number

$\mathrm{M}_{\text {aero }}=$ dimensional aerodynamic pitching moment

$\mathrm{M}_{\max }=\mathrm{ACM}$ maximum thrust moment

Midpt $=$ midpoint of a line

\footnotetext{
${ }^{1}$ Aerospace Engineer, Dynamic Systems and Control Branch, Mail Stop 308, Senior Member.

${ }^{2}$ Aerospace Engineer, Dynamic Systems and Control Branch, Mail Stop 308, Associate Fellow.

${ }^{3}$ Aerospace Engineer, Dynamic Systems and Control Branch, Mail Stop 308, Senior Member.

${ }^{4}$ Aerospace Engineer, Flight Dynamics Branch, Mail Stop 308, Member.

${ }^{5}$ Aerospace Engineer, Dynamic Systems and Control Branch Flight Dynamics Branch, Mail Stop 308,

${ }^{6}$ Aerospace Engineer, Flight Dynamics Branch, Mail Stop 308,

${ }^{7}$ Aerospace Engineer, Integrated GN\&C Analysis Branch, Member.

${ }^{8}$ Aerospace Engineer, Integrated GN\&C Analysis Branch.
} 


$\begin{array}{ll}\mathrm{qbar} & =\text { dynamic pressure } \\ \mathrm{T}_{\max } & =\mathrm{ACM} \text { maximum thrust } \\ \mathrm{x}_{\mathrm{ACM}} & =\text { thrust vector } \mathrm{x} \text {-coordinate location } \\ \mathrm{x}_{\mathrm{CG}} & =\text { center of gravity } \mathrm{x} \text {-coordinate location }\end{array}$

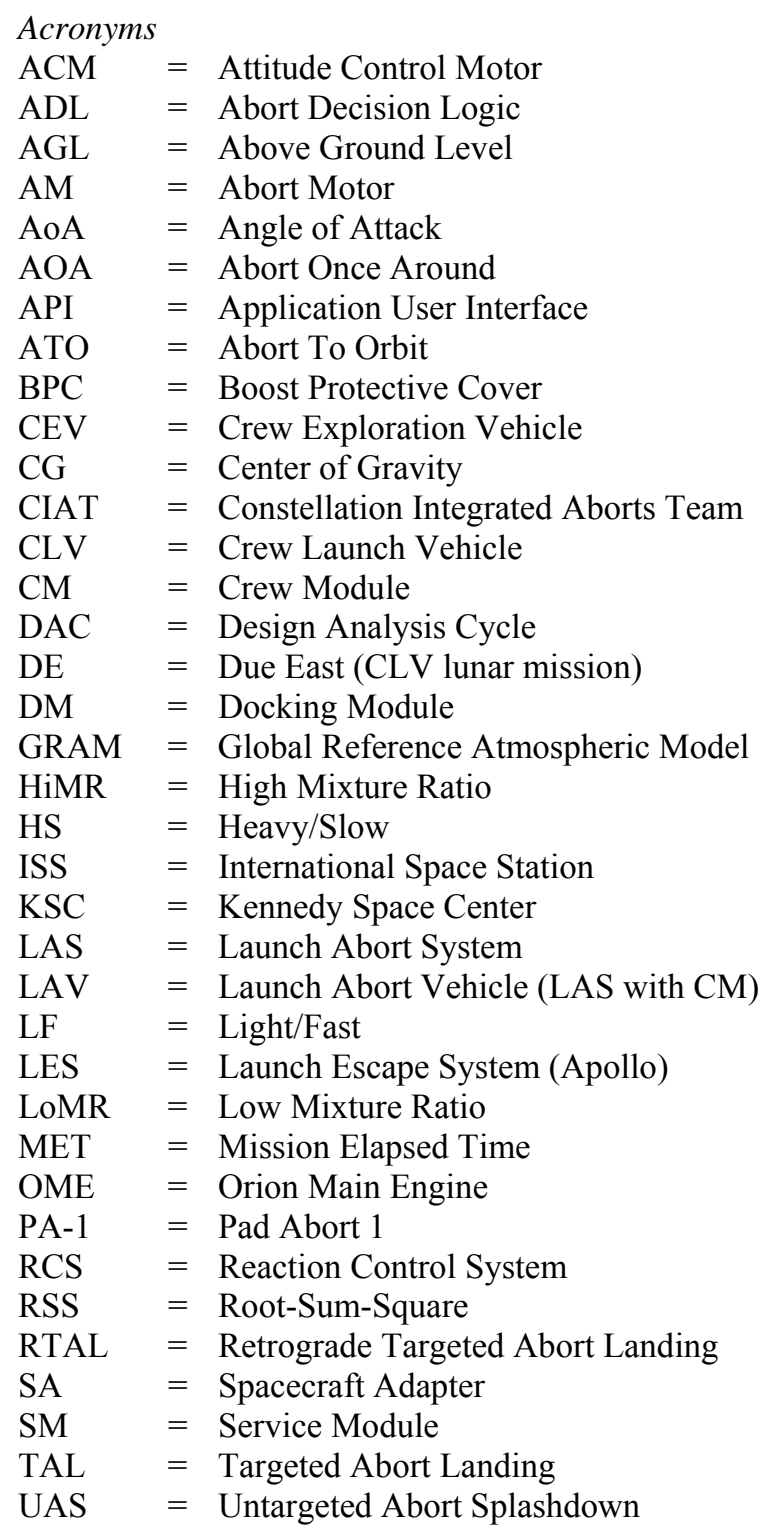

\section{Introduction}

NASA's Vision for Space Exploration calls for a long term exploration of the Moon, Mars, and beyond, with a focus on returning astronauts to the Moon by 2020. These goals require the development of a new spacecraft - the Orion Crew Exploration Vehicle $(\mathrm{CEV})^{1}$. One of the primary design drivers for the CEV is to ensure crew safety. CEV requirements specify that abort capability should be continuously available from the launch pad until the 
mission destination is reached. ${ }^{2}$ Aborts during the critical ascent flight phase require the design and operation of CEV systems to escape from the Crew Launch Vehicle (CLV) and return the crew safely to the Earth.

Ascent phase aborts are characterized by large changes in vehicle altitude, large amplitude attitude maneuvers, and large vehicle center-of-gravity movement; and pose significant engineering challenges. Several ascent abort modes are being designed and analyzed to accommodate the velocity, altitude, atmospheric, and vehicle configuration changes that occur during ascent ${ }^{3}$. These modes provide abort coverage extending from the launch pad until the CEV achieves a sustainable orbit. Analyzing these modes involves an evaluation of the feasibility and survivability of each abort mode and an assessment of the abort mode coverage using the current baseline vehicle design. Factors, such as abort system performance, concept of operations, crew load limits, thermal environments, crew recovery, and vehicle element disposal are investigated to determine if the current vehicle requirements are appropriate and achievable ${ }^{4,5,6}$.

Abort from the launch pad to early in the flight of the CLV second stage are performed using the Launch Abort System (LAS). During this type of abort, the LAS abort motor is used to pull the Crew Module (CM) safely away from the CLV and Service Module (SM). LAS aborts are characterized by high aerodynamic loads induced by low altitude maneuvers and high accelerations caused by the launch abort motor.

This paper presents an overview of the CEV in Section II and an overview of the LAS ascent abort mode in Section III. Three key LAS abort analysis methods and results are then discussed in Section IV and the paper concludes in Section V.

\section{Orion Crew Exploration Vehicle}

The Orion Crew Exploration Vehicle (CEV) is based on a design similar to the Apollo program's Command and Service Modules and is composed of four main elements: the Launch Abort System, the Crew Module, the Service Module, and the Spacecraft Adapter, as shown in Fig. 1. ${ }^{7}$ The Launch Abort System (LAS) provides a reliable abort capability for aborts that occur within the atmosphere. The Crew Module (CM) provides a safe habitable volume for the crew during launch, spaceflight, and return from the International Space Station (ISS) or Moon. The Service Module (SM) provides additional resources necessary to support the primary mission, including power and maneuvering capability. The SM is also used to provide abort capability for exo-atmospheric aborts. The Spacecraft Adapter (SA) provides the interface between the spacecraft and the Crew Launch Vehicle (CLV). The term Launch Abort Vehicle (LAV) will be used to refer to the LAS and CM together, prior to jettison of the LAS.

As shown in Fig. 2, the LAV consists of an Abort Motor to provide the CLV to LAV abort separation function, an Attitude Control Motor (ACM) to provide attitude and rate control, and a jettison motor for CM to LAS separation. The ACM consists of a throttle-able solid rocket system. The LAS also provides a Boost Protective Cover (BPC) that shields the CM from debris and the aero-thermal environment during ascent.

In addition to its nominal mission functions, the SM also provides abort capability during the high altitude portions of the CLV launch profile. The SM contains two propulsion systems: the Service Module Orion Main Engine (SM OME) (used for large maneuvers, such as orbit changes and re-entry) and the Reaction Control System (RCS) (used for fine control, such as proximity operations and docking, as well as providing a backup for the OME) ${ }^{8}$. For LAS assisted aborts, the LAV separates from the $\mathrm{SM}$ at the SM-CM plane. In the event of a CLV problem after nominal jettison of the LAS, the SM OME and RCS thrusters can be fired to maneuver the CEV to safety.

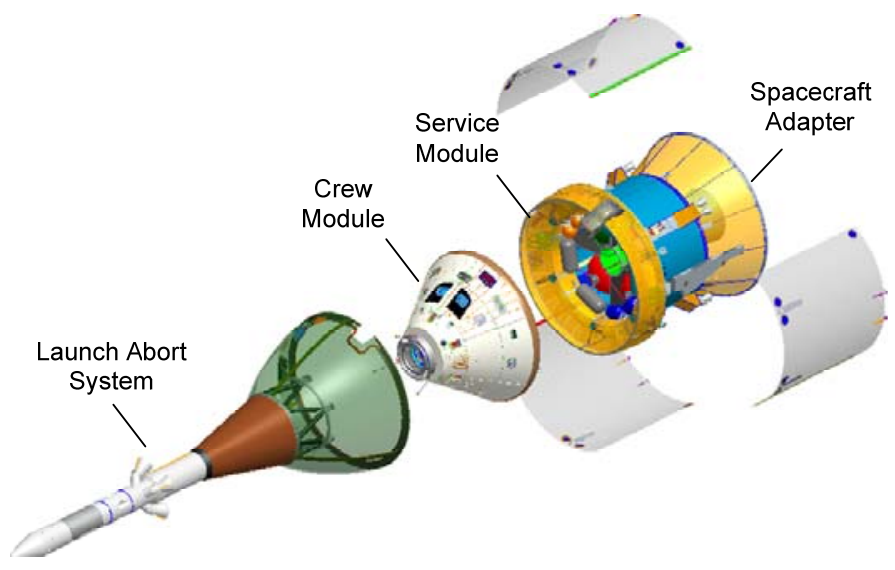

Figure 1. Orion Crew Exploration Vehicle. 


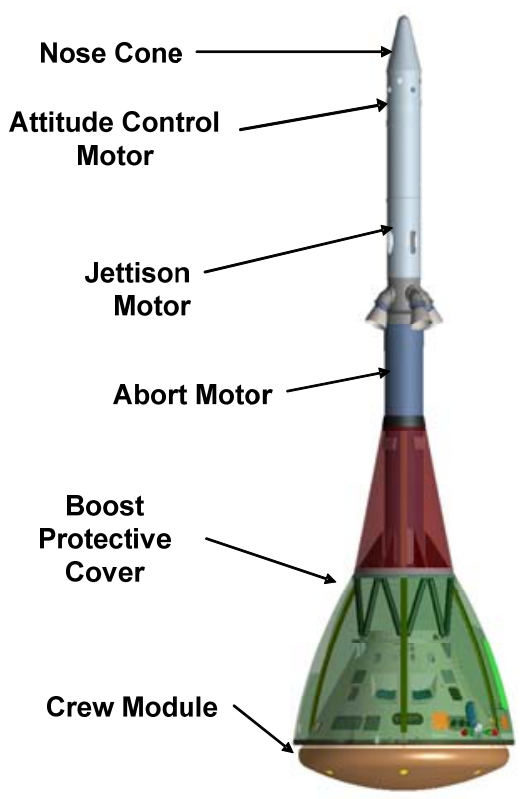

Figure 2. Elements comprising the Orion Launch Abort Vehicle (LAV).

\section{CEV Ascent Abort Mode Overview}

The CEV system is required to provide a launch abort capability from the launch pad after $\mathrm{CM}$ hatch closure throughout the CLV ascent until orbital insertion. The possible reasons for an ascent abort fall into one of three general categories: (1) a partial or total loss of CLV propulsion, (2) a loss of CLV control, or (3) a systems failure, either on the CLV or CEV, which results in the inability to safely achieve orbit. Several abort modes are required to accommodate the velocity, altitude, atmospheric, and vehicle configuration changes that occur during ascent. These modes provide abort coverage extending from the launch pad until the CEV achieves a sustainable orbit.

Mode I aborts, also referred to as Launch Abort System (LAS) aborts, are performed using the LAS and remain a viable option until the LAS is nominally jettisoned early in the CLV second stage burn. During Mode I aborts, the LAS abort motor is used to pull the Crew Module (CM) away from the CLV and Service Module (SM). Mode I aborts are characterized by high aerodynamic loads induced by low altitude maneuvers and high accelerations caused by the launch abort motor. LAS aborts may be commanded via the ground-based health management system, the on-board CEV

Abort Decision Logic (ADL), the crew, or ground personnel.

Mode II aborts, also referred to as Untargeted Abort Splashdown (UAS) aborts, do not utilize the LAS. Instead, the CLV upper stage engine is shut down and the SM Reaction Control System (RCS) is used to provide adequate clearance between the CLV and CEV. Once the CEV is sufficiently far away from the launch vehicle, the CM separates from the SM, and then the CM is maneuvered for a guided re-entry, and descends using parachutes to a safe landing location.

Mode III aborts, commonly known as Targeted Abort Landings (TAL), are triggered by late second stage failures where the CEV trajectory is modified via a targeted SM OME burn followed by a CM guided entry to a target landing site. The goal of these trajectory control efforts is to select a landing site that maximizes the chances of crew survival and recovery, while also meeting crew loads and SM thermal constraints.

The last type of abort mode is Mode IV, which is an Abort To Orbit (ATO). This mode describes cases where an abort is performed following a premature shutdown of the upper stage when the SM has sufficient capability to achieve a safe orbit insertion and de-orbit burn. ${ }^{8}$

\section{Launch Abort System Aborts}

Launch Abort System (LAS) aborts, or Mode I aborts, are performed using the LAS and remain a viable option until the LAS is nominally jettisoned at a pre-designated point during the flight of the CLV second stage. The current CEV design uses an active LAS approach that stabilizes and reorients the capsule during the launch abort maneuver using an Attitude Control Motor (ACM) in the escape tower (Figure 3). This approach differs from the passive approach used on Apollo which relied upon the use of open-loop timers to control the abort event sequencing along with a passive canard that was deployed to reorient the vehicle to a heat-shield forward attitude following the escape motor firing and a brief coast period. The Apollo Launch Escape System (LES) ${ }^{9}$ is shown in Figure 4. The passive Apollo system required approximately 1,000 pounds of ballast to ensure aerodynamic stability during the escape motor firing and coast, and the passive canard system could produce tumbling in some portions of the envelope before settling into a heat-shield forward attitude.

The use of the active LAS on the CEV eliminates both the ballast required to ensure passive aerodynamic stability in the nose-forward orientation as well as the open-loop pitch motor weight included in the passive Apollo. A feedback control algorithm is currently under development that issues commands to the ACM system to stabilize, pitch, and reorient the LAV during the abort. The current concept controller uses a proportional/integral/derivative (PID) approach to control attitudes and rates of the LAV. 


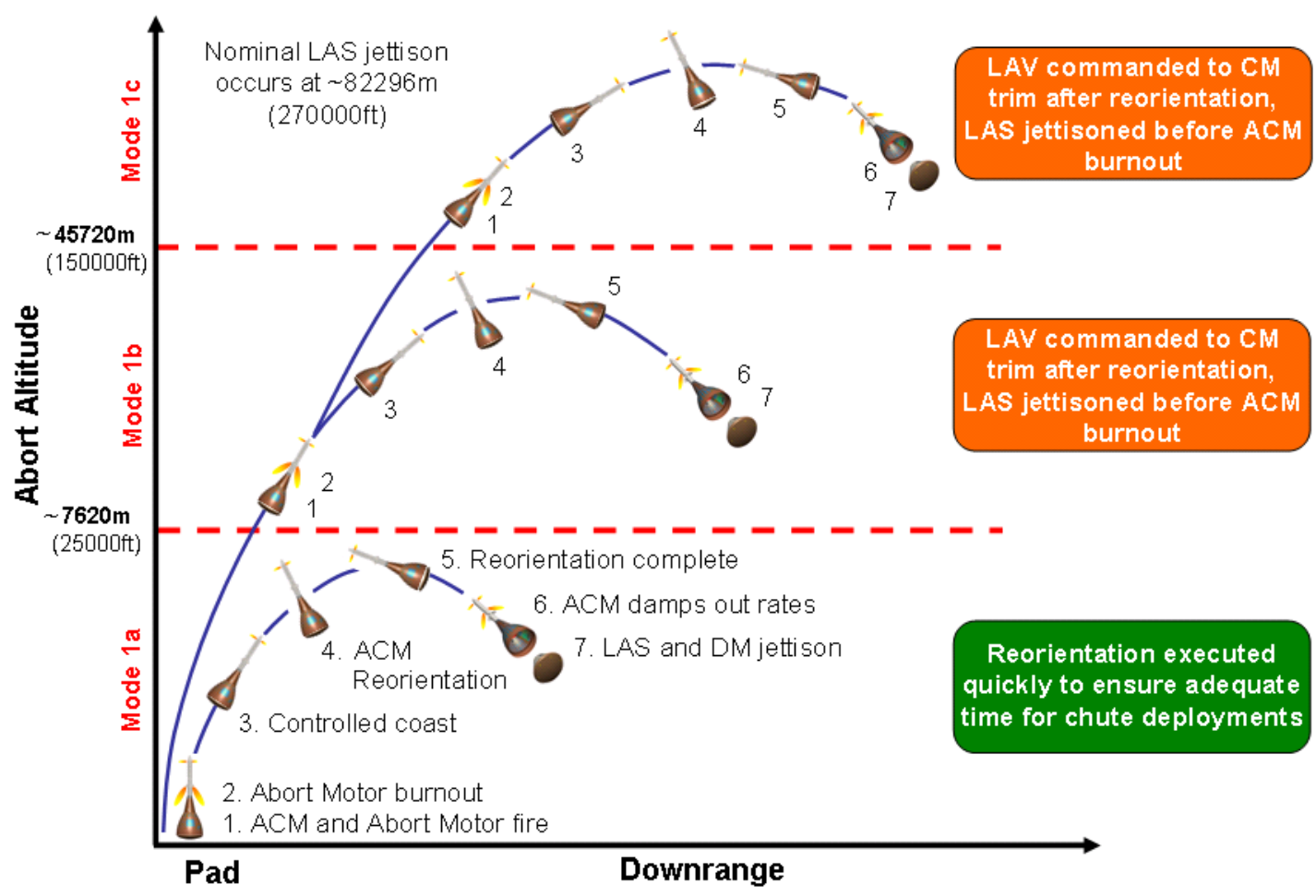

Figure 3. CEV Launch Abort System Abort Scenarios.

The concept of operation and event sequencing during an abort varies with altitude. The LAS aborts are divided into three regimes by altitude above ground level (AGL) at abort initiation as follows: Low: 0 to 25,000 feet; Mid: 25,000 feet to 150,000 feet; High: 150,000 feet to nominal LAS jettison (See Figure 3). The sequence of events during an abort is based on abort initiation altitude, time elapsed from abort initiation, and estimated dynamic pressure and Mach number.

Once an abort condition is detected, the abort motor and ACM are ignited. After the abort motor burns out (approximately 4 seconds), the vehicle continues on a controlled coast trajectory. The ACM provides the control during abort motor operation and coast to maintain nose-forward flight. The reorientation maneuver is initiated at an elapsed time between 8 and 15 seconds to orient the LAV with the heat-shield into the velocity vector, assuring proper attitude for parachute deployment and, from high altitudes, proper thermal protection system (TPS) orientation. The ACM is used to track the reorientation maneuver command. Following reorientation, the ACM is used to stabilize the LAV until the LAS is jettisoned from the CM using the Jettison Motor.

There is a water landing depth and impact velocity requirement that the trajectory must achieve at LAS jettison to ensure a successful chute deployment and water landing following a pad abort. To achieve these requirements in the

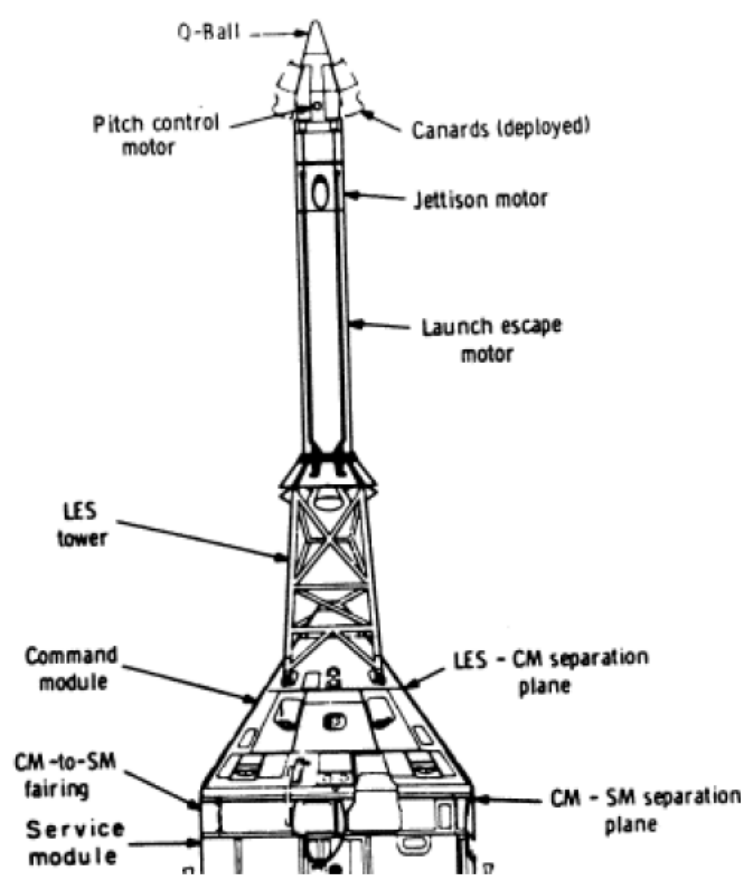

Figure 4. Apollo Launch Escape System (Ref. 9). 
case of a pad abort, a pitch maneuver must be performed during the abort motor burn, so that the abort motor thrust is directed along the proper trajectory. The brevity of the abort motor firing period means that the pitch maneuver must be fairly abrupt if it is to be effective in directing the abort trajectory. The high rates and attitudes of the pitch maneuver in the pad abort regime make it one of the more demanding of all the abort cases in terms of the authority required from the ACM. Sufficient control authority must be provided to ensure that the LAV does not tumble due to abort motor thrust misalignments or aerodynamic uncertainties during this portion of the abort.

\section{LAS Ascent Abort Design Analysis}

Launch Abort System abort performance analysis involves an evaluation of the feasibility and survivability of a LAS abort using the current baseline vehicle design. These studies and design trades are being conducted so that more informed decisions can be made regarding the vehicle requirements, design, and operations. ${ }^{10}$ Examples of three analyses being used to assess LAS abort flight dynamic performance are: Launch Abort System Performance Analysis, Launch Abort System Static Controllability Envelope Analysis, and Launch Abort Vehicle Tip-Off Conditions Due to Crew Launch Vehicle Failures and Dispersions. Summaries for these three analyses are described in the sections below.

\section{A. Launch Abort System Performance Analysis}

This study examined the performance and controllability of the current Launch Abort System abort configuration (LAV), which includes an extended aerodynamic faring that shields the CM from debris and the aero-thermal environment during ascent. The controllability analysis includes a survey of non-dispersed aborts conducted at regular intervals along a nominal CLV ascent trajectory, as well as Monte Carlo dispersed aborts conducted from the launch pad, maximum dynamic pressure, and high altitude abort flight conditions.

The analysis was performed using the ANTARES simulation with a rotating, oblate Earth model. ${ }^{11}$ ANTARES is a code that uses the Trick Simulation Environment for defining and tying together various dynamical and environmental models, written in either C or FORTRAN, for 6-DOF simulation execution. The simulation also used the GRAM-99 atmospheric model, including GRAM-99 winds for mid and high altitude abort regimes. ${ }^{12}$ For pad aborts, the $1840 \mathrm{KSC}$ winds profile set was used. All simulations were run from initiation of the abort up to the drogue chute deployment. Simulation runs were terminated at drogue chute deployment, and several flight condition performance metrics were applied at this point. The forward bay cover (FBC) jettison and drogue chute deployment events were not modeled in the simulations. Neither the potential for recontact of the CM and the LAS during the LAS jettison event, nor transient forces and moments that may be imparted to the CM as a result of the LAS jettison were included in this study. Since all simulation runs were terminated at the initiation of the drogue deployment, no portion of the drogue or main chute deployment sequence was included in this study.

The simulation activity was divided into two parts. The first part took the form of an abort trajectory survey, where a series of aborts were simulated from regularly-spaced altitude intervals (1000 feet), from 300 feet up to 300,000 feet. No dispersions were applied in this survey; all model and system parameters were maintained at their nominal values. Also, no winds were applied in the survey simulations. The controller performance was then evaluated in terms or attitude, attitude rate, Mach number and dynamic pressure throughout the abort maneuver.

For the second part, the simulation analysis consisted of Monte Carlo dispersions for three abort scenarios: 1) at the pad; 2) at maximum CLV dynamic pressure; and 3) at high altitude (i.e., at altitudes near the expected LAS jettison point from a nominal ascent trajectory). The perturbations in this Monte Carlo analysis included the following system parameters: 1) LAS abort motor mis-alignment and thrust level output (due to temperature variation); 2) LAS ACM and abort motor points of action; 3) aerodynamic data; and 4) LAS and CEV mass properties (i.e., mass, inertia and center of mass locations); 4) variations in atmospheric properties (provided by GRAM-99); and 4) variations in initial abort flight conditions. Performance metrics were then assessed for each abort scenario.

\section{Relevant Performance Metrics}

The performance metrics that are prescribed for the abort are intended to ensure that controlled flight is maintained, that structural constraints are observed with respect to aerodynamic loading, and that the CM is left in a flight dynamic condition that is appropriate for successful drogue deployment following the LAS jettison event. These performance metrics take the form of limits that are applied to allowable variations in angle of attack, sideslip, body rates, dynamic pressure, and Mach Number values at certain points along the simulated abort trajectory. Some key performance metrics being assessed are as follows: 
Angle of attack $(\alpha)$ and sideslip angle $(\beta)$ must satisfy:

- Maintaining nose-forward flight prior to start of reorientation maneuver

- At drogue deployment (2-seconds after LAS jettison),

140 degrees $\leq \alpha \leq 210$ degrees

-40 degrees $\leq \beta \leq 40$ degrees

Magnitudes of rotational roll, pitch, and yaw body rates $(p, q, r)$ were evaluated over a 2 -second interval prior to drogue chute deployment, with success criteria:

Max. $|q|,|r| \leq 40 \mathrm{deg} / \mathrm{sec}$

Max. $|p| \leq 80 \mathrm{deg} / \mathrm{sec}$

Max. $\left(q^{2}+r^{2}\right)^{1 / 2} \leq 40 \mathrm{deg} / \mathrm{sec}$

Dynamic pressure (qbar) and Mach number $(\mathrm{M})$ at drogue deployment must satisfy:

$10 \leq$ (qbar) $\leq 160$ pounds per square foot $(\mathrm{psf})$

$\mathrm{M} \leq 0.9$

Throughout the maneuver, an aerodynamic loading constraint is applied:

$($ qbar $) *\left(\alpha_{\text {tot }}\right) \leq 17,000$ psf-degrees

\section{Results/Discussion}

The results for the controllability analysis are presented in this Section. The abort trajectory survey results are presented first, followed by Monte Carlo analysis of a pad abort.

\section{Abort Trajectory Survey}

The results of the abort trajectory survey are shown in this section. For this analysis, a series of aborts were simulated from regularly-spaced altitude intervals (1000 feet) (from 300 feet up to 300,000 feet) along a nominal CLV trajectory. The presentation of the data has been divided into three categories, based upon initial abort altitude regimes: Low, from 0 to 25,000 feet; Mid, from 25,000 to 150,000 feet; and High, from 150,000 feet. and above.

Figures $7-9$ show the aerodynamic angle, body rate, and Mach and dynamic pressure time histories for the Low abort regime.
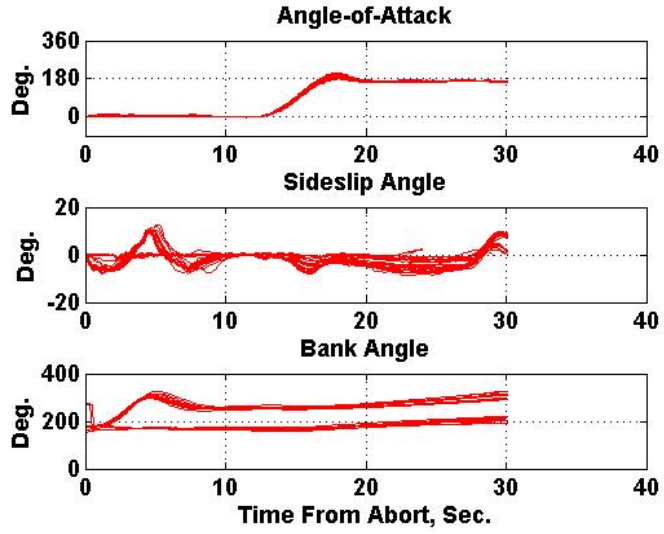

Figure 7: Aerodynamic angles in Low abort regime.
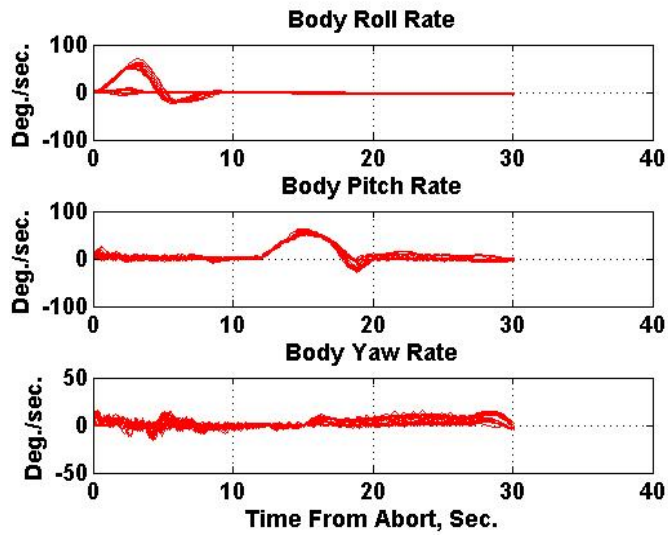

Figure 8: Body rates in Low abort regime.

From the angle of attack plot in Figure 7, it can be noted that all the trajectories in this Low abort regime successfully perform the abort reorientation maneuver to the heatshield forward attitude. The sideslip and bank angle time histories indicate a switch in their behavior as the abort altitude increased. For initial abort altitudes 
above 9,000 feet, the LAV rolled during the Abort motor burn; and the sideslip angles peaked above 10 degrees. However, these sideslip excursions settled before the start of the reorientation maneuver. The attitude and body rates post-LAS jettison were well within acceptable levels for drogue chute deployment for all abort trajectories in this Low altitude regime. Similarly, the Mach and dynamic pressure data, shown in Figure 9, indicate that these values also satisfied their respective drogue deployment performance metrics.

Figure 10 plots the altitude versus the total range (as measured from the abort initiation for each trajectory) values at LAS jettison. Note that the effect of the sideslip excursion starting at aborts above 9,000 feet and below 17,000 feet tended to reduce net ranges.

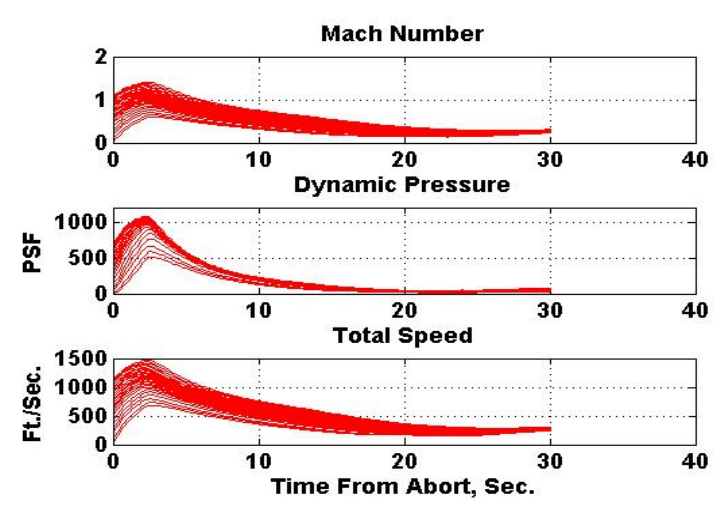

Figure 9: Mach number, dynamic pressure, and velocity in Low abort regime.

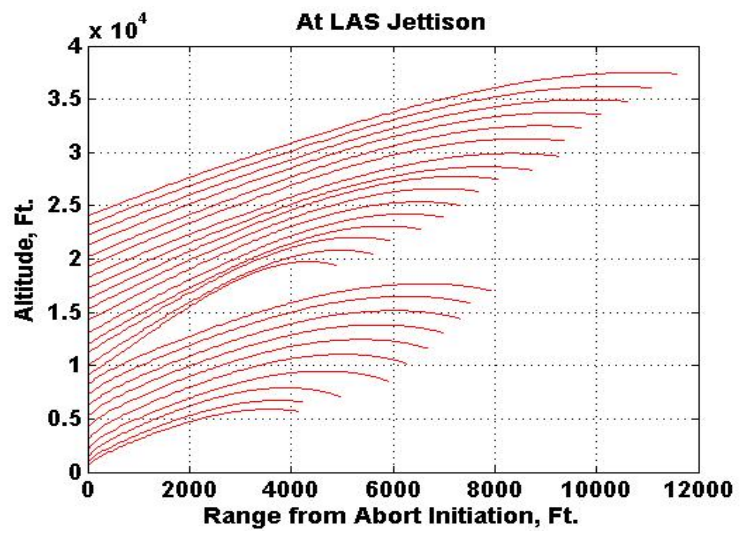

Figure 10: Altitude vs. range from abort initiation.

The abort survey performance results for the mid altitude abort regime are presented in Figures $11-13$. The reorientation maneuver generates large amounts of overshoot for many of the lowest altitude aborts in this regime due to the high dynamic pressure at which the maneuver is initiated. The reorientation must begin no later than 15 seconds into the abort in order to stabilize the LAV in the heat-shield forward attitude before the ACM propellant is depleted. At high dynamic pressure, larger aerodynamic torques are produced as the LAV breaks out of the noseforward attitude generating much higher pitch rates during the maneuver, resulting in more overshoot of the commanded reorientation attitude. Larger sideslip angle excursions are also apparent during the reorientation, again owing to the higher dynamic pressure. However, even with the higher aerodynamic moments encountered during this high dynamic pressure abort regime, the ACM settled the LAV to acceptable attitude and attitude rate levels around the LAS jettison point.
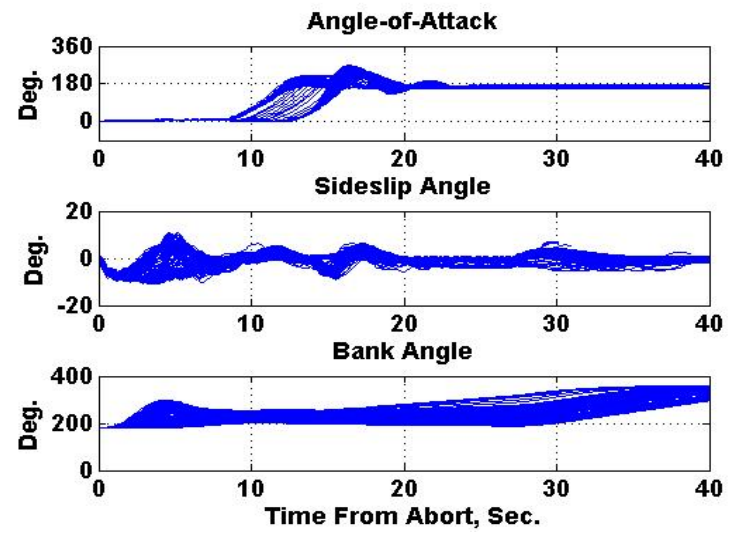

Figure 11: Aerodynamic angles in Mid abort regime.
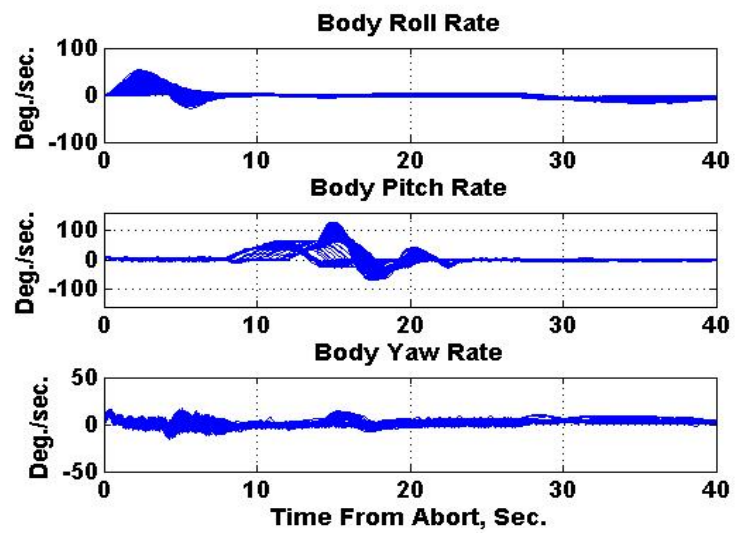

Figure 12: Body rates in Mid abort regime. 


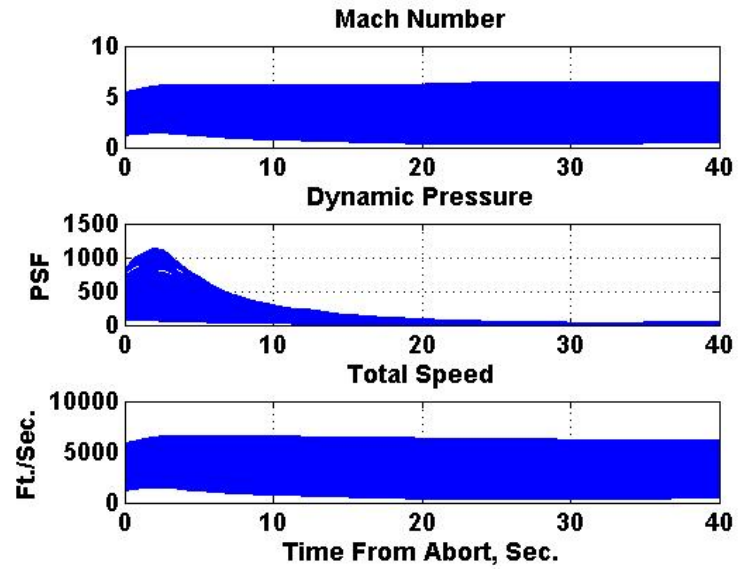

Figure 13: Mach number, dynamic pressure, and velocity in Mid abort regime.

Finally, Figures $14-16$ show the High altitude abort regime performance results. Far less variation in the dynamic response of the system is observed for aborts in this regime due to the much lower dynamic pressures at which the aborts are conducted. Figure 16 shows that dynamic pressure was less than $60 \mathrm{psf}$ throughout all aborts in this regime. The angle of attack time histories show that the reorientation maneuver starts at 8 seconds, since the dynamic pressure criteria was already less than $100 \mathrm{psf}$ at the beginning of the reorientation time window. Positive control of rate and attitude is exhibited throughout the abort since the ACM control authority is sufficient to overcome all aerodynamic torques.

The build-up of body rates in the roll and yaw axes after the LAS jettison point may be attributed to the CM RCS activity. In the ANTARES simulation, 3-axes CM RCS control was active post-LAS jettison, switching to the entry controller. Further analysis must be done to determine the reason for this RCS activity, as the vehicle attitude and rates appear to be well within acceptable levels pre-LAS jettison.
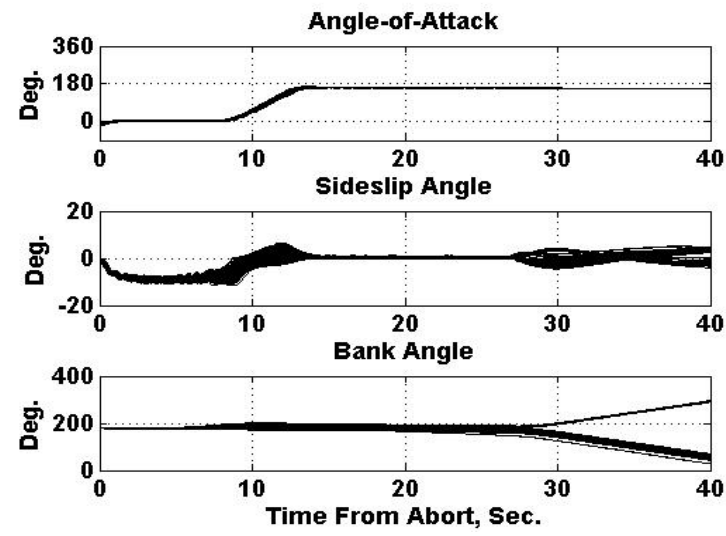

Figure 14: Aerodynamic angles in High abort regime.
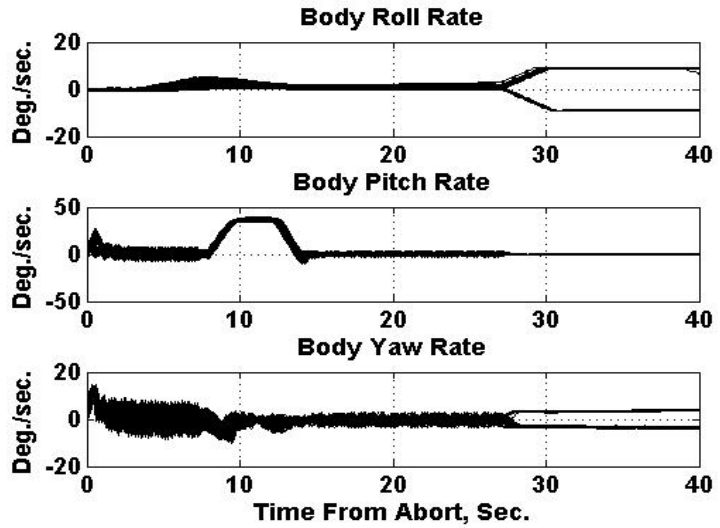

Figure 15: Aerodynamic angles in High abort regime. 


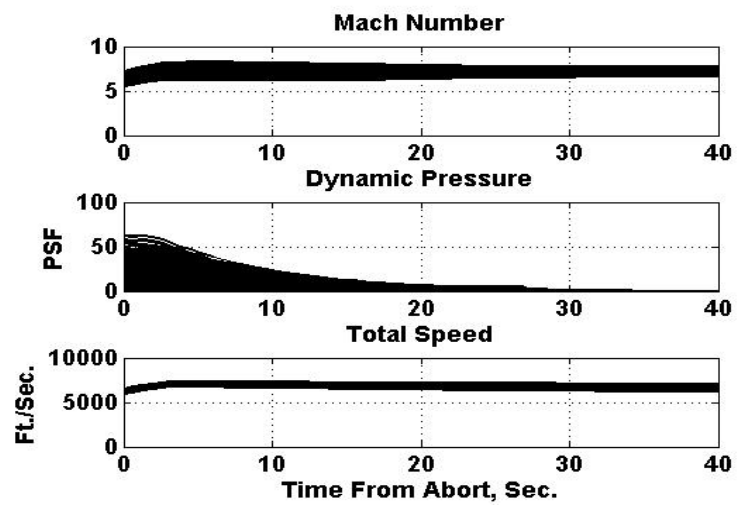

Figure 16: Mach number, dynamic pressure, and velocity in High abort regime.

\section{Monte Carlo Simulation Results - Pad Abort}

Simulation time histories produced by the 1840-run Monte Carlo dispersions for the KSC pad abort are shown in Figure 17. The angle of attack time histories illustrate that no runs tumbled or failed to reorient properly. The maximum sideslip angles are around 10 degrees in these cases, and this does not appear to jeopardize the success of the reorientation maneuver or the ability to achieve a successful LAS jettison.
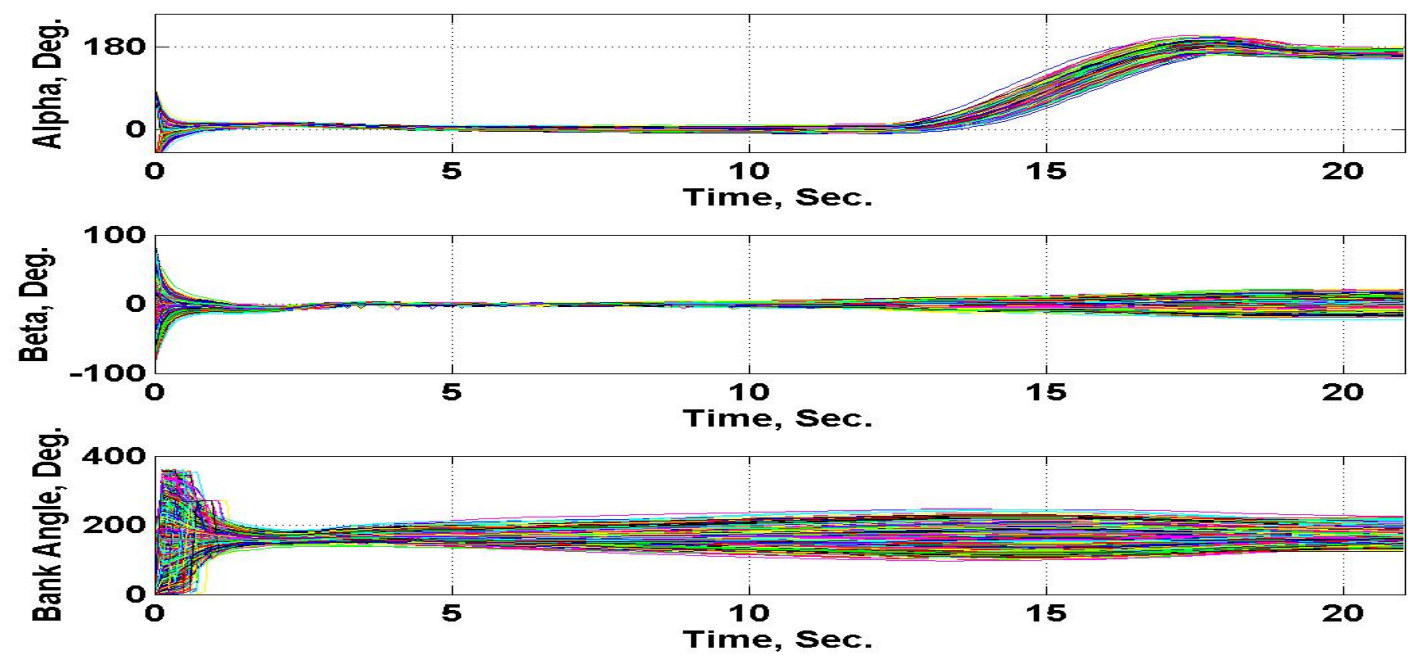

Figure 17: Pad abort Monte Carlo aerodynamic angle time histories.

Shortly after the LAV reorientation maneuver in a pad abort, the LAS is jettisoned at an elapsed time of 21 seconds, and the crew module $(\mathrm{CM})$ experiences approximately two seconds of open-loop flight prior to drogue deployment. Tolerances on angle of attack and sideslip of the CM have been specified for successful drogue deployment. The allowable angle of attack range is between 140 and 210 degrees, and the sideslip range is between +40 and -40 degrees. Dispersion results for the angle of attack and sideslip of the CM at the approximate time of drogue deploy are shown in Figure 18, with the prescribed tolerances indicated as a green box. 


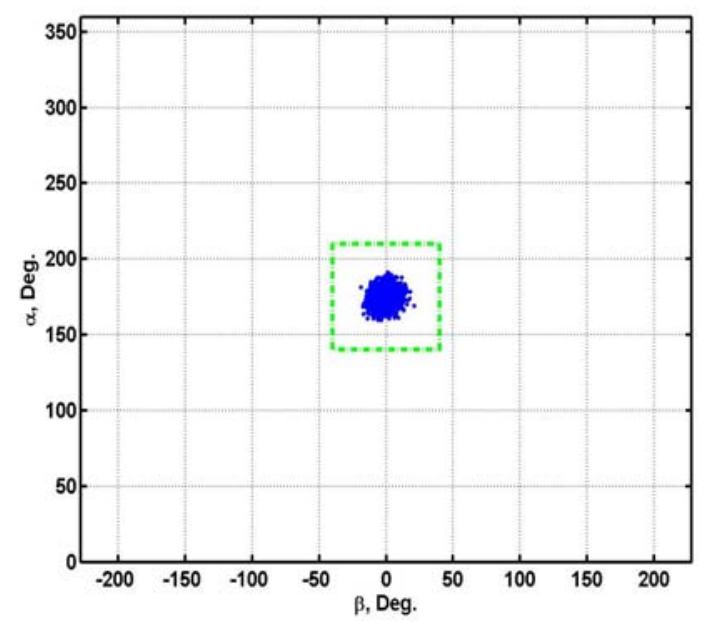

Figure 18: Pad abort dispersion plots of angle-ofattack vs. sideslip of $\mathrm{CM}$ at the approximate drogue deployment point.

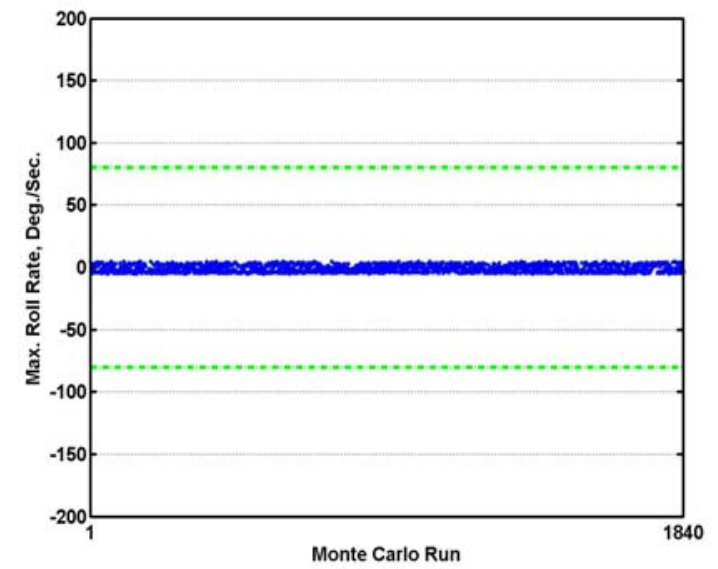

Figure 20: Pad abort maximum CM roll rates observed during the time period between LAS jettison and drogue deployment.

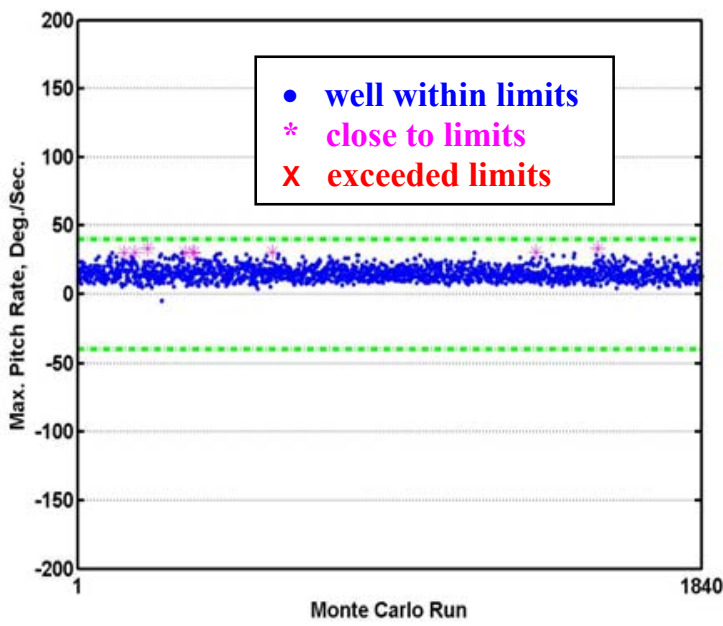

Figure 19: Pad abort maximum $\mathrm{CM}$ pitch rates observed during the time period between LAS jettison and drogue deployment.

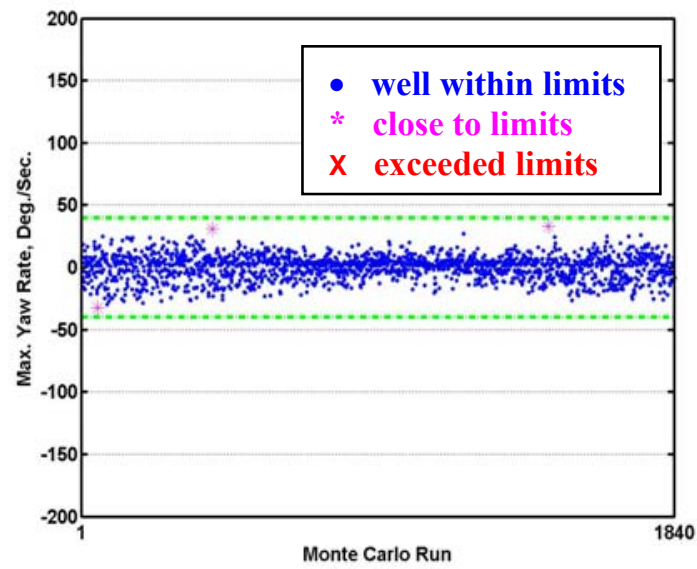

Figure 21: Pad abort maximum CM yaw rates observed during the time period between LAS jettison and drogue deployment.

Acceptable ranges for angular rates of the CM have also been defined for the period of flight between LAS jettison and drogue deployment. The magnitudes of pitch and yaw rates must be less than 40 degrees per second $(\mathrm{deg} / \mathrm{sec})$, and roll rate magnitudes must be less than $80 \mathrm{deg} / \mathrm{sec}$. Figure 19 shows the largest pitch rates that occurred for each run between LAS jettison and drogue deployment. Most runs were well with the pitch rate limit. Only seven runs have rates close to the limit (i.e., within $10 \mathrm{deg} / \mathrm{sec}$ ). Figure 20 shows the analogous plot for roll rates of the CM. Roll rates were well within acceptable limits. Recall, the maximum allowable roll rates were set at $\pm 80 \mathrm{deg} / \mathrm{sec}$, instead of the $\pm 40 \mathrm{deg} / \mathrm{sec}$ prescribed for pitch and yaw rates. Figure 21 shows maximum yaw rate excursions. Only three out of 1840 rates approached the designated drogue chute rate limits.

The last two drogue chute condition performance metrics, which concerns the dynamic pressure and Mach number values, are plotted in Figure 22. No excursions beyond the limits of these parameters were encountered. 
The aerodynamic loading metric is examined next. A constraint on aerodynamic loading is placed on the LAV flight condition expressed as a limit on the product of dynamic pressure (qbar) and total angle of attack. The maximum allowable product is 17,000 psf-deg. Figure 23, shows the product time traces during the first ten seconds of the abort maneuver. The peak values occurred after 2.5 seconds (towards the end of the Abort Motor burn). None of the peak values exceeded the dynamic pressure total angle of attack product limit.

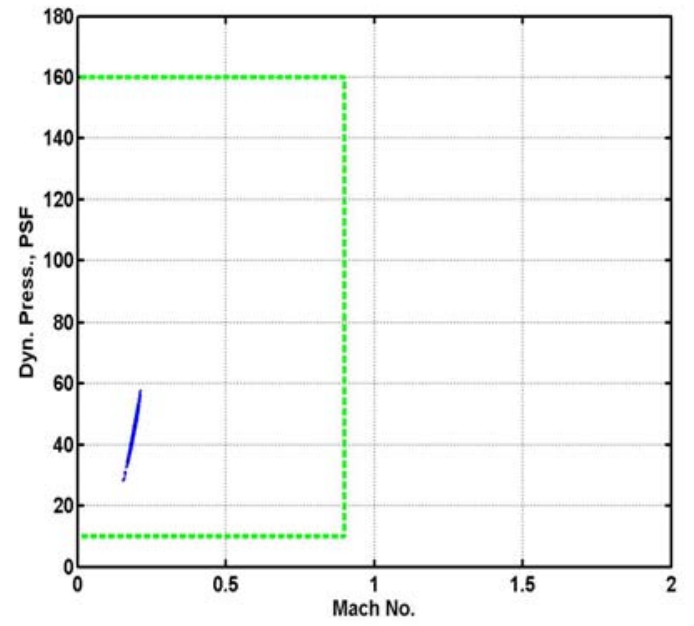

Figure 22: Pad abort dispersion plots of dynamic pressure vs. Mach number of $\mathrm{CM}$ at the approximate drogue deplovment point.

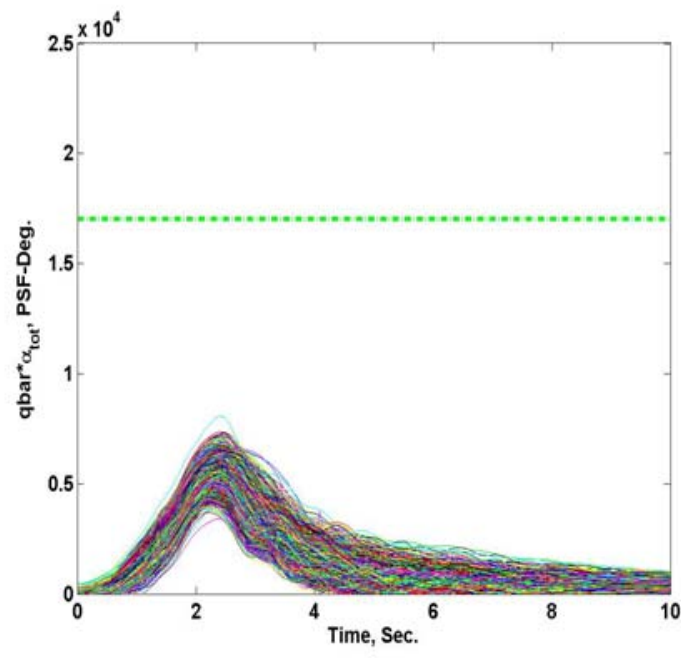

Figure 23: Pad abort dispersion plots of dynamic pressure $x$ total angle-of-attack of the LAV for the first ten seconds of the abort.

\section{B. Launch Abort System Static Controllability Envelope Analysis}

A static controllability tool was created to provide a visual means of assessing the controllability of the LAV. The tool consists of a series of MATLAB ${ }^{\circledR}$ m-files and uses simulation time histories and the LAV aerodynamics database to produce animated plots of the vehicle's angle of attack versus dynamic pressure trajectory plotted over regions identified as stable or unstable and controllable or uncontrollable. The tool is being used to investigate the flight dynamics characteristics of two LAV design configurations at various abort conditions. A preliminary analysis of the two configurations provides explanations for behaviors seen in simulated trajectories and a first look at comparing the controllability of the two vehicle configurations. The configurations considered in this study included a passive canard deployed to assist in the LAV reorientation maneuver (maneuver to transition from nose forward to heat-shield forward flight).

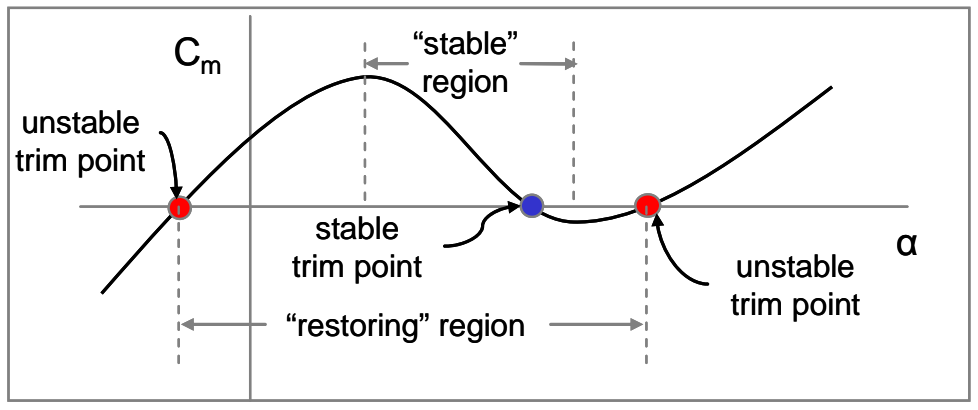

Figure 24. Illustration of definitions of stable and restoring regions for pitching moment (about the center of gravity) versus angle of attack. 
The controllability plots include two key regions - stability and controllability. The two regions are defined in the pitch plane only and are determined using static aerodynamic coefficients. There are two approaches to determining the limits of the pitch-plane stable region. The first is to find a stable trim point and the range of angle of attack around that trim point where $\mathrm{C}_{\mathrm{m}_{\alpha}}$ is negative. An alternate definition of this region exists and can be useful in some cases. For each angle of attack, if the pitching moment is such that is returns the vehicle to a noseforward state, then the vehicle is defined as stable. If the vehicle's tendency is to orient to a heatshield-forward state, then the vehicle is defined as unstable. The two methodologies produce different stable region boundaries, but both are acceptable as long as the chosen definition is understood. For the analysis presented here, the latter-defined "stable" region is referred to as a "restoring" region. A difficulty in defining the restoring region is that it can only be used for showing nose-forward stability. When the vehicle performs the reorientation maneuver to a heatshield-forward position, the definition of restoring would have to change. Since one of the goals of this tool is to show the reorientation of the vehicle to a heatshield-forward position, the former definition of stability is chosen for this tool. Figure 24 illustrates the described methods to determine whether a moment is stable or restoring.

Three options for displaying

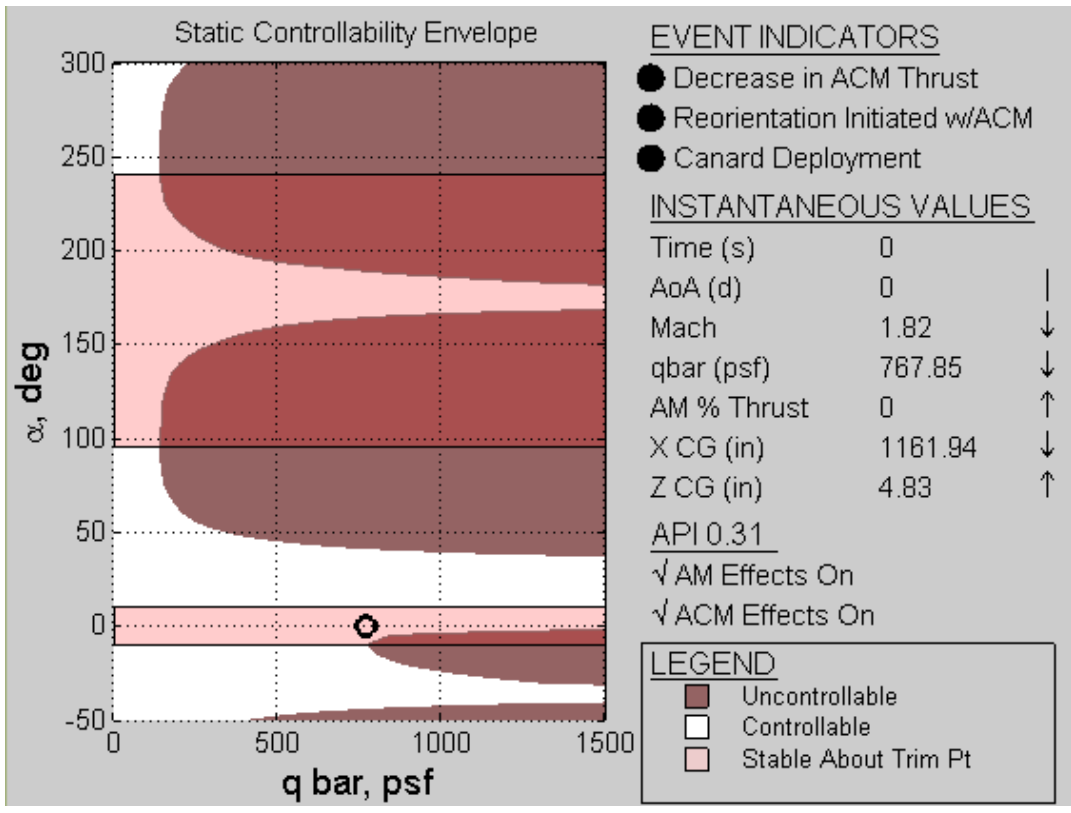

Figure 25a. Example plot with stable region defined by negative $C_{m a}$ about a stable trim point.

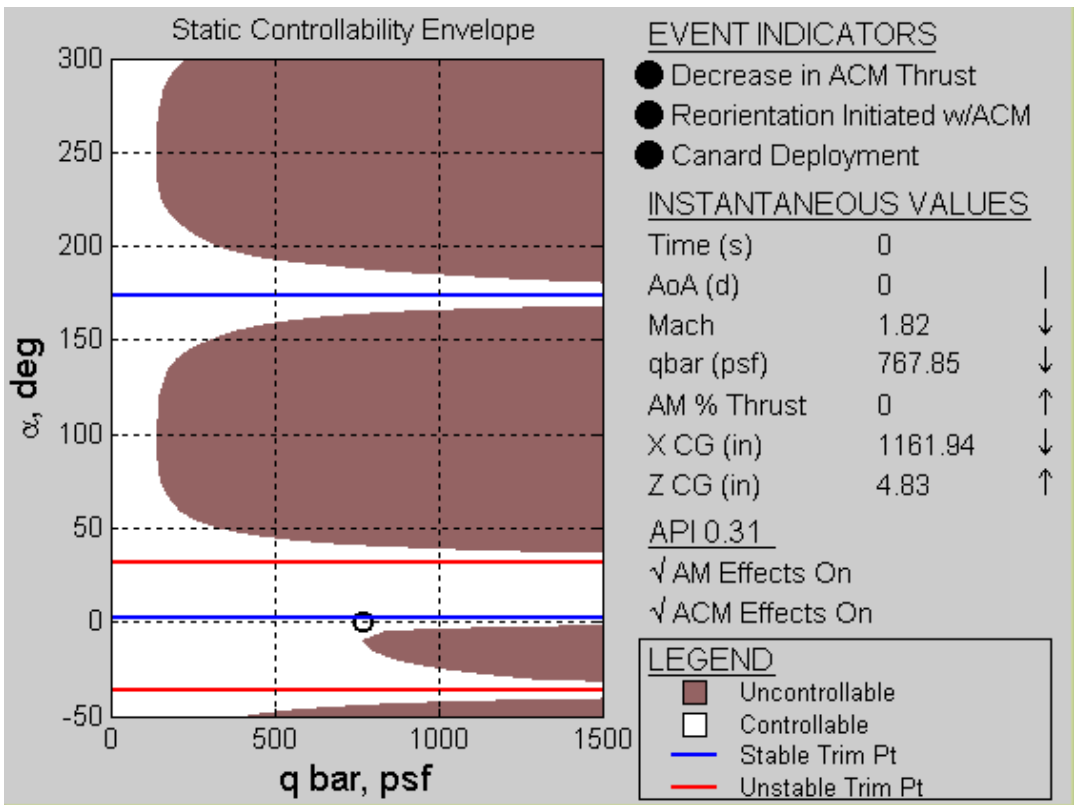

Figure 25b. Example plot with stable and unstable trim points shown by lines. stable regions are provided by this tool: 1) no stability display (to emphasize controllability region), 2) stable region defined by negative $C_{m \alpha}$ about a trim point, or 3) stable and unstable trim points shown by horizontal lines on the plot. Examples of the latter two options are shown in Figures 25a and 25b, respectively. More details on the figures are described later in this section.

The first step in determining the controllable region is to determine the dimensional aerodynamic pitching moment (about the center of gravity) over the angle of attack - dynamic pressure space. An example of the absolute value of the computed dimensional aerodynamic pitching moment is shown in Figure 26. 
The ACM maximum thrust moment is found by multiplying the maximum thrust that the ACM can produce at a given time by the distance from the center of gravity of the vehicle to the ACM thrust vector at a given time, shown in Eq. (1).

$$
M_{\max }=T_{\max }\left(x_{A C M}-x_{C G}\right)
$$

Comparing the maximum thrust moment to the aerodynamic moment over the angle of attack - dynamic pressure space determines whether the vehicle is statically controllable or uncontrollable. If the aerodynamic moment is larger than the ACM maximum moment, then the vehicle is uncontrollable, and vice versa.

The controllability plot is animated over the span of the LAV's trajectory from abort initiation until tower jettison and includes the entire angle of attack range that the vehicle experiences during flight. A stillframe image of the tool output after completing a simulated pad abort is shown in Figure 27. In the animations produced by the tool, there are several important factors that influence the regions. The vehicle center of gravity, Mach number, canards, ACM jet interaction effects and abort motor jet interaction effects are all influencing the pitching moment. The vehicle center of gravity and ACM maximum thrust over time are influencing the ACM maximum thrust moment. The abort motor and ACM jet interaction effects affect the controllability envelope because they may increase or decrease the pitching moment for certain angles of attack. The white lines in the uncontrollable region appear after the initial decrease in maximum ACM thrust from 7,000 to 2,500 pounds at 6 seconds after abort initiation and show where the controllable region boundary would be if the ACM was still producing the maximum 7,000 pounds of thrust.

The event indicators are another feature of the tool that consist of circles that are initially black and turn a different color when a particular event happens. The color change of the circle corresponds to a change in trajectory line color on the plot, as can be seen in Figure 27. The noted events

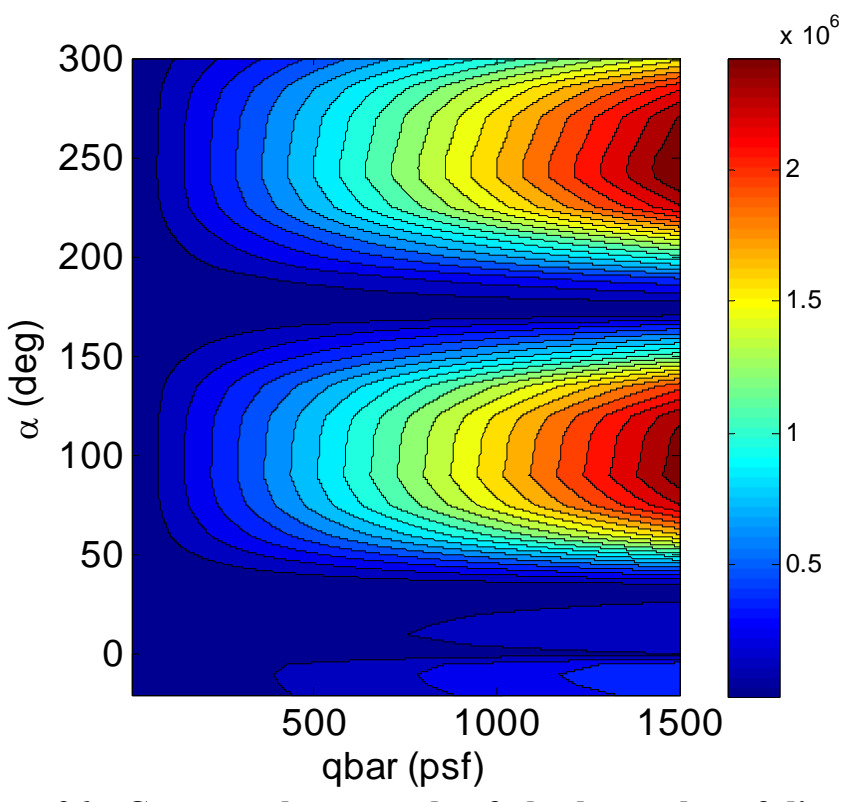

Figure 26. Contour plot example of absolute value of dimensional aerodynamic pitching moment over angle of attack - dynamic pressure space.

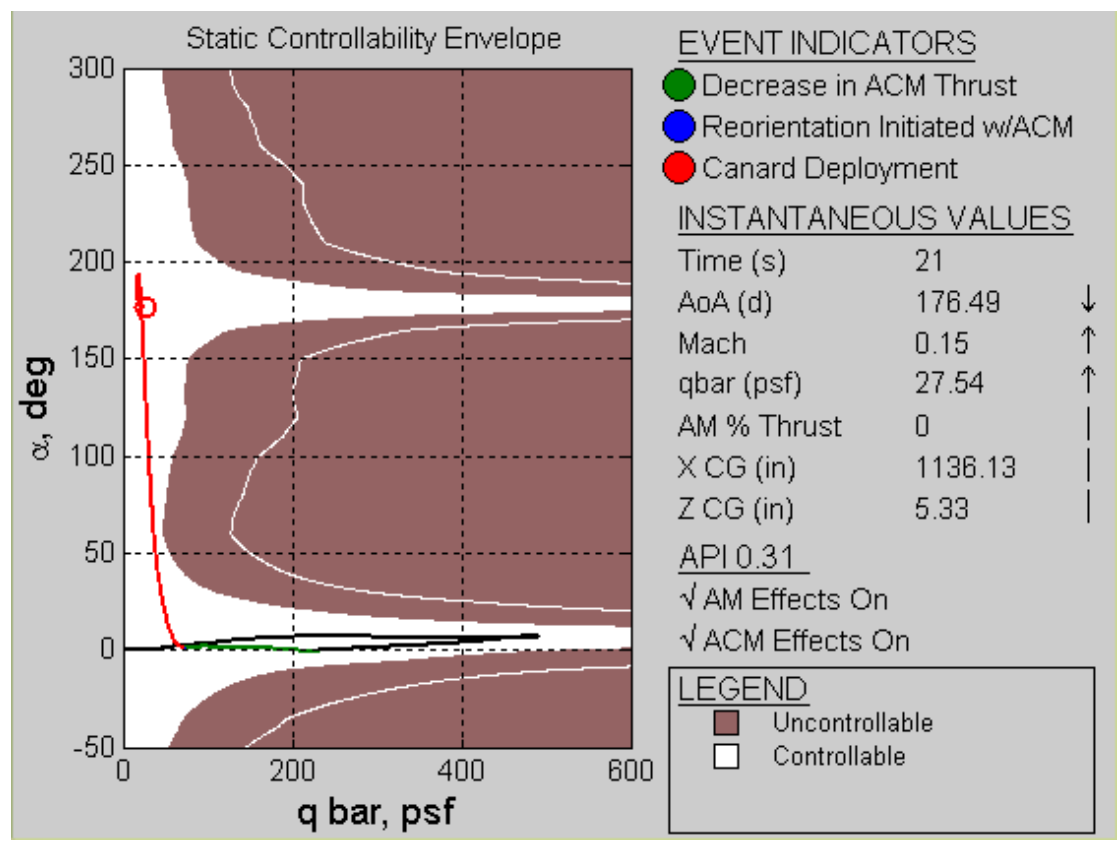

Figure 27. Still frame image of animated controllability tool. 
are the decrease in ACM thrust, initiation of reorientation with the ACM, and deployment of the canards. The reorientation initiated with the ACM and the deployment of the canards often happens at the same time, as can be seen by the red color overlapping the blue on the trajectory line for the pad abort case in Figure 27. The circle on the trajectory line is where the vehicle is currently at during the animation, while the line left behind shows where the vehicle has been.

Instantaneous values of time in seconds, angle of attack (AoA) in degrees, Mach number, dynamic pressure (qbar) in psf, abort motor thrust as a percent of maximum thrust (AM \% Thrust), and center of gravity location in both $\mathrm{x}$ and $\mathrm{z}$ directions in inches are shown. The arrows next to each of those values indicate whether the particular value is increasing or decreasing at a particular time in the vehicle's trajectory. A straight line indicates that the value is constant.

The last section appearing on the right side of the controllability envelope plot includes the version of the aerodynamics database (0.31 in this example), as well as check marks to indicate that the abort motor and ACM jet interaction effects are included in the determination of the controllability regions. Cross symbols appear if those effects are not included. As shown in Figure 25 , the legend updates according to the option of stability the user has chosen to view.

The controllability envelope tool was used to assess the flight dynamics of both the operational LAS configuration and the Pad Abort 1 (PA-1) flight test vehicle configuration. The PA-1 flight test configuration is based on an earlier baseline configuration that has higher drag than the current configuration. A preliminary analysis of these two configurations shows not a large difference in their respective controllable regions. Both configurations stay within the controllable region for the nominal pad abort case. The largest difference is observed in the angle of attack versus dynamic pressure trajectories for the two configurations plotted on top of the controllable regions and is seen best in the maximum dynamic pressure abort regime. Figures $28 \mathrm{a}$ and $28 \mathrm{~b}$ show the last frame of the animations for a maximum dynamic pressure abort for both configurations.

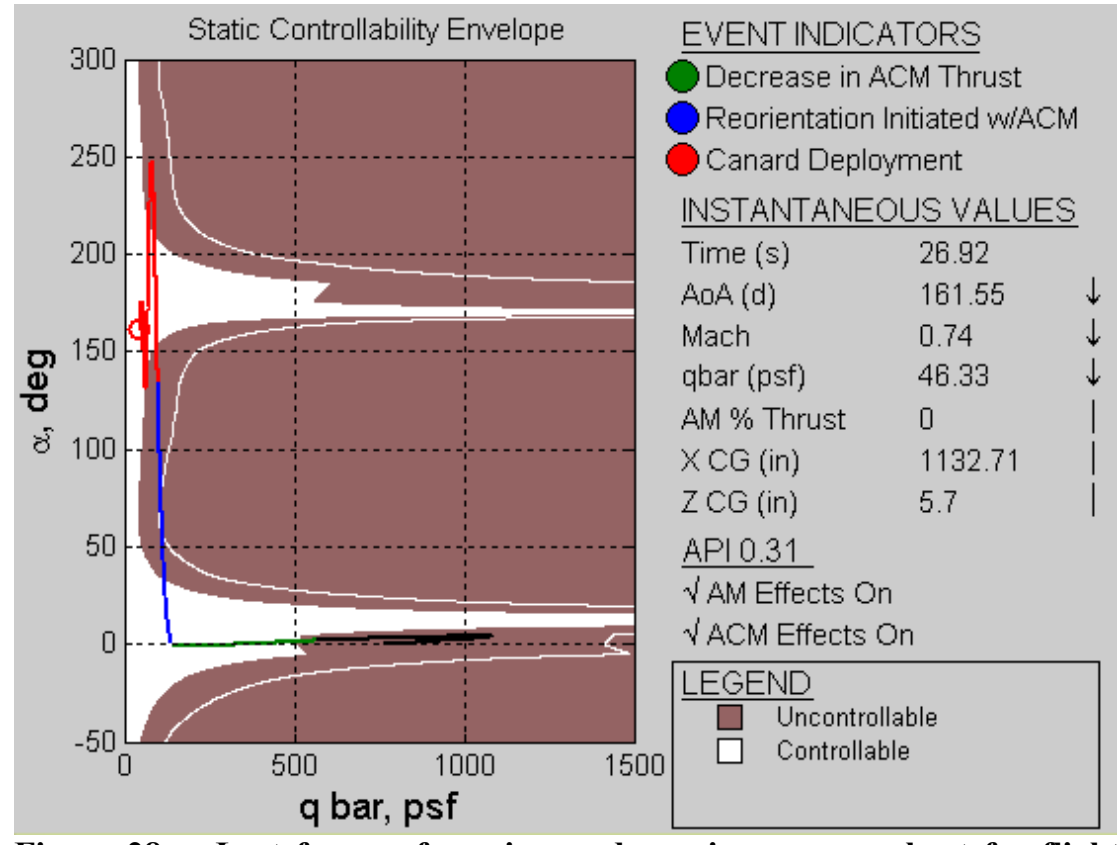

Figure 28a. Last frame of maximum dynamic pressure abort for flight test vehicle configuration.

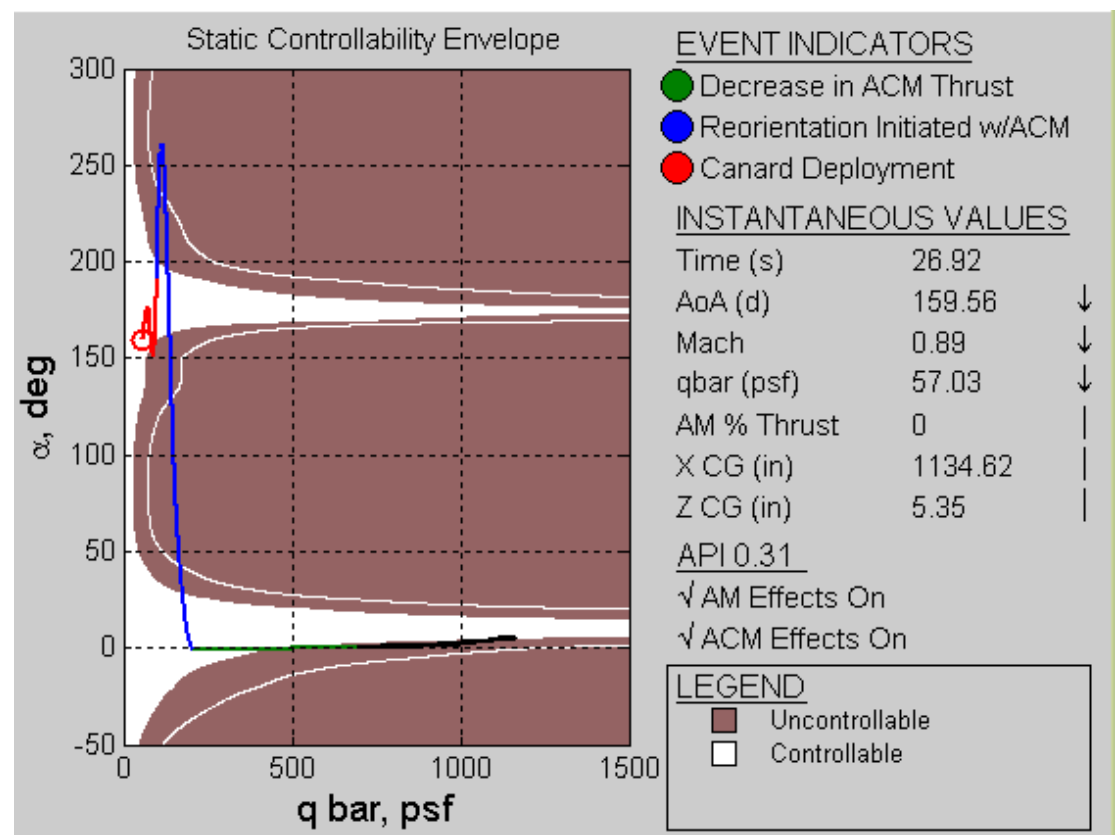

Figure 28b. Last frame of maximum dynamic pressure abort for operational vehicle configuration. 
For the maximum dynamic pressure abort case, both configurations enter the uncontrollable region during the vehicle reorientation. This explains why neither vehicle can reorient at its commanded rate in the maximum dynamic pressure abort regime leading to overshoots of the commanded angle of attack. There is not enough ACM control authority to overcome the static aerodynamic moment. However, both vehicles have a stable trim point and a large statically stable region for the heatshield-forward attitude that is keeping them from tumbling even when they are in a statically uncontrollable state.

There is also a noticeable difference in canard deployment time. The canards are commanded to deploy when the dynamic pressure of the vehicle falls below 100 psf. The drag modeled in version 0.31 of the aero-database is lower for the operational vehicle configuration than the flight test vehicle configuration. This difference between the two configurations causes the flight test vehicle to slow down faster than the operational vehicle, leading to an earlier deployment of the canards, prior to reorientation, for the flight test vehicle. The operational vehicle deploys its canards after the vehicle has completely reoriented. During the reorientation, the drag difference also causes the operational vehicle to go farther into the static uncontrollable region leading to greater overshoot of the commanded angle of attack as can be seen in Figure 28.

The tool can also be used to investigate the cause of an unsuccessful abort. For example, an abort may fail because the vehicle unintentionally tumbles after initiating an abort and before the vehicle was intended to reorient. The controllability tool can show whether the vehicle entered an uncontrollable and unstable region during this event.

\section{Launch Abort Vehicle Tip-Off Conditions Due to Crew Launch Vehicle Failures and Dispersions}

The LAV is required to be capable of successfully aborting from the launch pad up until the LAS is nominally jettisoned at a pre-designated point during the flight of the CLV second stage. This study examined dispersed CLV trajectory data sets (dispersed Crew Launch Vehicle (CLV) ascent trajectories and CLV failure trajectories.) to determine the range of potential LAV tip-off conditions (initial conditions at LAS abort initiation). The results of this study are used as abort initial conditions to assess LAS abort performance, such as in the abort trajectory survey study described earlier In the following, the abort envelopes are presented, along with the methods and assumptions used to obtain them.

The most current set of CLV trajectories analyzed is labeled Design Analysis Cycle 2, Revision 4 (DAC-2 Rev4). There are nine no-failure dispersed CLV trajectories. Each dispersion set consists of 2,000 runs. The simulations were performed with the NASA Marshall Space Flight Center (MSFC) 6 degree-of-freedom simulation MAVERIC (Marshall Aerospace Vehicle Representation in C).

The nine no-failure trajectory sets analyzed are as follows:

1) Heavy/slow due east February mission with launch at close of launch window and high second stage mixture ratio (HS DE Feb Close HiMR)

2) Heavy/slow due east February mission with launch at close of launch window and low second stage mixture ratio (HS DE Feb Close LoMR)

3) Heavy/slow due east February mission with launch at opening of launch window (HS DE Feb Open)

4) Heavy/slow International Space Station (ISS) February mission with launch at opening of launch window and high second stage mixture ratio (HS ISS Feb Open HiMR)

5) Heavy/slow ISS February mission with launch at opening of launch window and low second stage mixture ratio (HS ISS Feb Open LoMR)

6) Light/fast due east February mission with launch at close of launch window ( $L F$ DE Feb Close)

7) Light/fast due east July mission with launch at opening of launch window (LF DE July Open)

8) Light/fast ISS August mission with launch at close of launch window (LF ISS Aug Close)

9) Light/fast ISS August mission with launch at opening of launch window (LF ISS Aug Open)

Due east indicates that the CLV trajectory is designed for a lunar mission, while ISS indicates that the trajectory is designed to go to the ISS. Heavy/slow and light/fast denote the weight and speed of the vehicle. The second stage mixture ratio refers to the CLV J2X engine mixture ratio of fuel to oxidizer being lower or higher than average. The nominal LF ISS Aug Open trajectory parameters for the portion of flight where the LAS is still attached to the integrated stack are presented in Figure 29. 

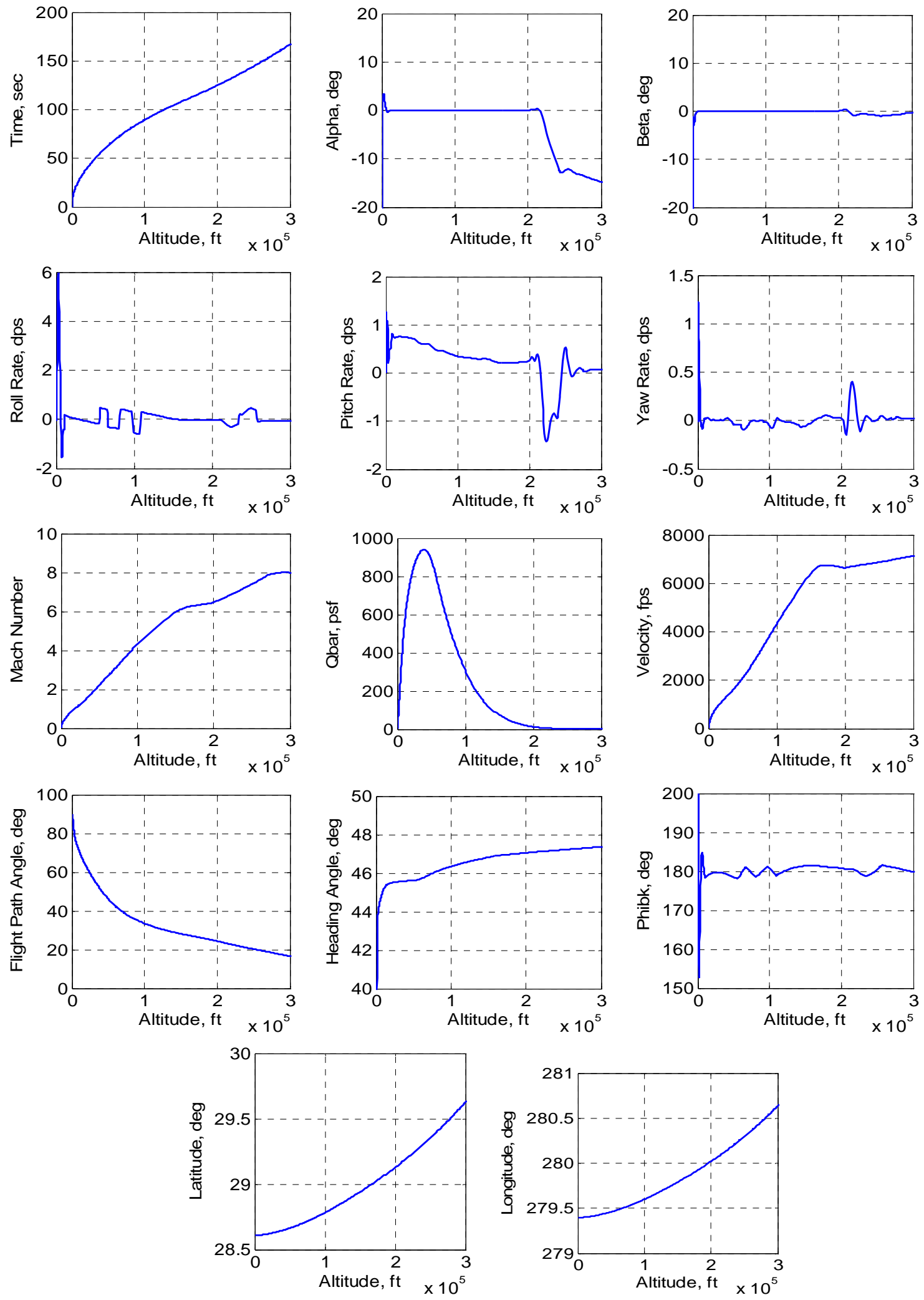

Figure 29. Nominal LF ISS Aug Open CLV trajectory while LAS is attached. 

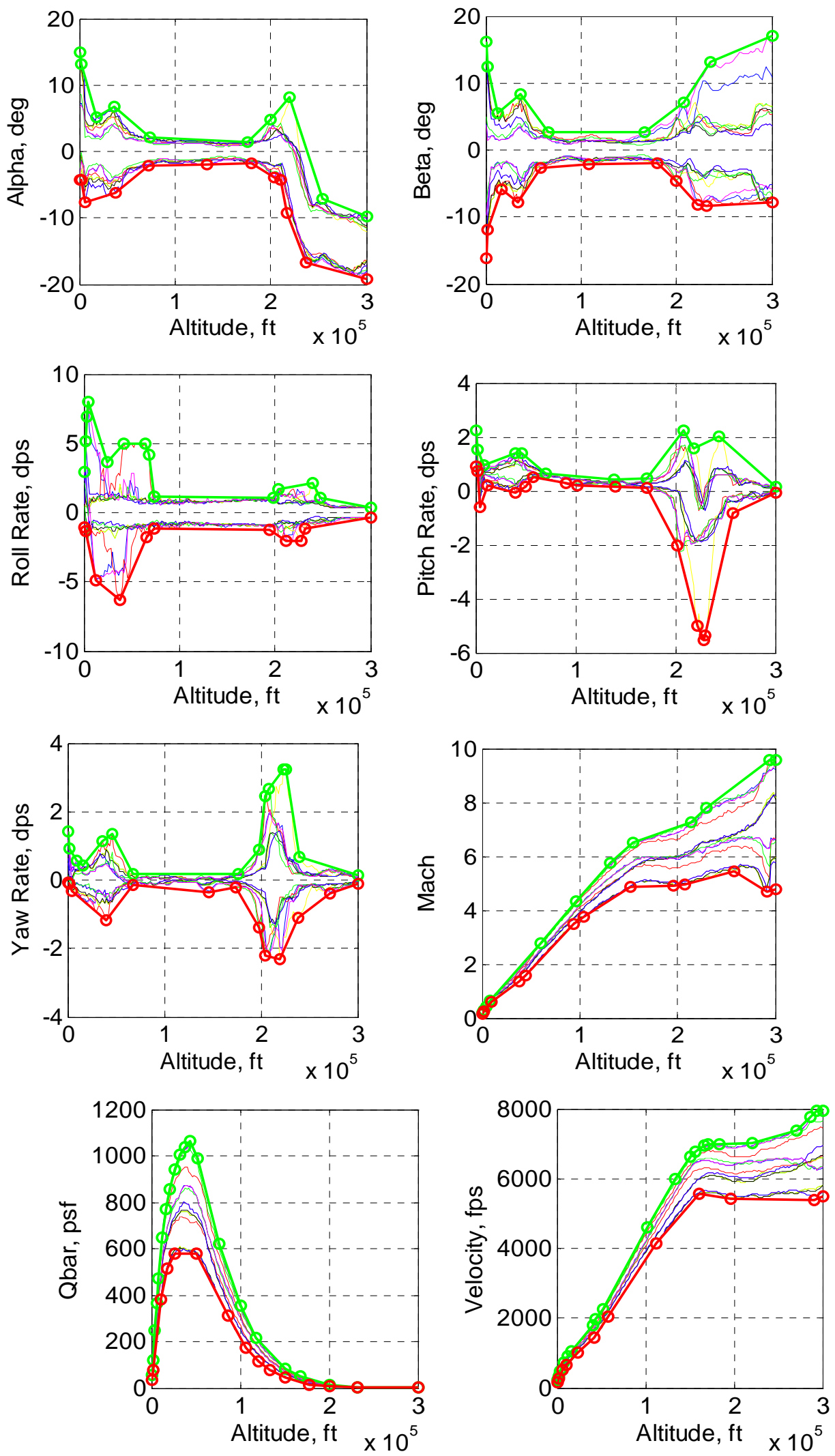

Figure 30. Abort Envelopes for Monte Carlo No-Failure Trajectories.

18

American Institute of Aeronautics and Astronautics 
For the no-failure dispersed trajectories, boundaries were placed around each of the nine sets by finding the minimum and maximum value of a particular condition at a given altitude step along the trajectories. Since the trajectory data is at a constant time step, not altitude step, each condition occurs at a different altitude value. Since there were not any discontinuities between each time step for the conditions along the trajectories, the conditions were interpolated to get values at desired altitude steps. Then the minimum and maximum values were found at each altitude step along the trajectories. In addition, an envelope was created around the conditions to include the trajectories from all nine Monte Carlo sets. To minimize the number of points defining each envelope, the envelopes were produced by hand-picking points from plots of all the trajectories.

Envelopes were produced for the following abort conditions versus altitude: time, angle of attack, sideslip angle, roll rate, pitch rate, yaw rate, Mach number, dynamic pressure, velocity, flight path angle, heading angle, bank angle, latitude, and longitude. The velocity is the relative magnitude accounting for winds.

The maximum and minimum limits for some of the conditions for each of the nine sets of dispersed CLV trajectories are shown in Figure 30. The bold green and red lines are the boundaries encompassing all nine of those. The green line represents the maximum of all of the dispersed data, and the red line represents the minimum of all of the dispersed data.

The failure trajectory data was produced by simulations performed with MAVERIC. Simulated failures were engine nozzle actuators that failed in either the rock or tilt directions. Rock and tilt are 45 degrees offset with respect to the body axes, so a failure in either affects both pitch and yaw of the vehicle. The actuators locked (stuck) into one of three positions: in place, at null, or at hard-over. Locked at hard-over denotes that the actuator becomes locked at its maximum deflection level of \pm 4 degrees.

There are eleven sets of Monte Carlo trajectories with 2000 simulated failures that were initiated randomly throughout a given time frame. Six of these examined the maximum dynamic pressure (qbar) time frame, and five examined the entire first stage of flight. Nine of these eleven dispersed sets are based on the LF ISS Aug Open CLV mission. The types of failures simulated in these dispersions are shown in Table I. The other two, one each from the maximum qbar time frame and the entire first stage, are based on the LF DE Feb Close CLV mission. These two dispersion sets simulate a lock in place rock and tilt actuator failure.

Table I. Failure types for dispersed CLV trajectory sets.

\begin{tabular}{|l|l|}
\hline Maximum $\bar{q}$ Time Frame & First stage of flight \\
\hline Lock in place rock actuator & Lock in place rock actuator \\
\hline Lock in place rock and tilt actuator & Lock in place rock and tilt actuator \\
\hline Lock at null rock actuator & Lock at null rock actuator \\
\hline Lock at hard-over rock actuator & Lock at hard-over rock actuator \\
\hline Lock at hard-over tilt actuator & \\
\hline
\end{tabular}

The LAS is responsible for aborting from the CLV in the event of a failure until nominal LAS jettison, which includes first stage burn and the first 30 seconds of second stage burn. However, failure trajectory data is only available for the first stage of the CLV, which corresponds to an altitude of about 210,000 feet depending on the mission. The abort conditions considered thus far begin at an altitude of 2,000 feet and do not include pad abort conditions. Little abort condition information due to failures is available below 2,000 feet.

An abort is triggered when the CLV exceeds any one of the following parameters, provided as a function of altitude: Y body acceleration, $\mathrm{Z}$ body acceleration, angle of attack, angle of sideslip, roll rate, pitch rate, or yaw rate. These values were provided by the Constellation Integrated Aborts Team (CIAT) and are based on maximum values from the nine no-failure CLV Monte Carlo analyses with high wind gusts. A $20 \%$ margin was added for measured parameters and a 30\% margin was added for angle of attack and angle of sideslip since they are calculated onboard and not measured.

The approximate abort latency time

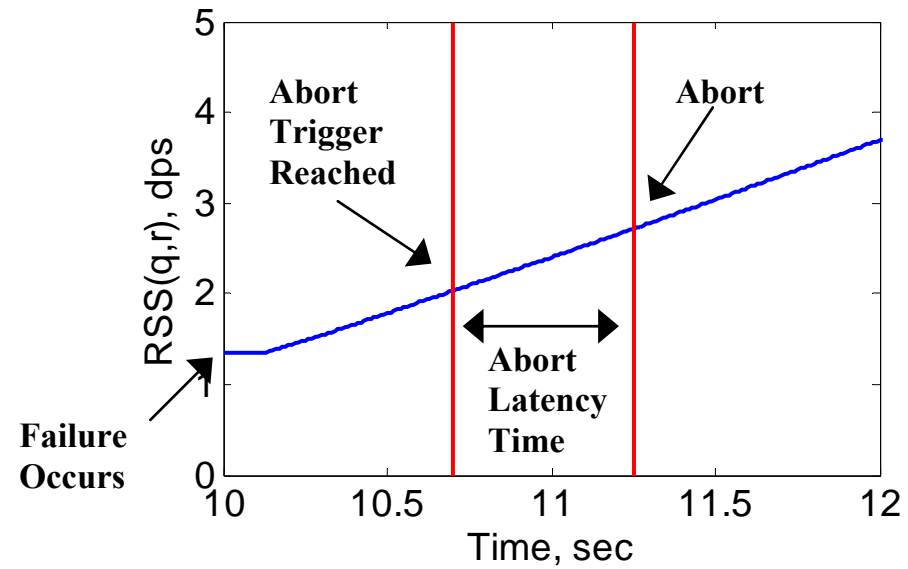

Figure 31. Timeline of events for CLV failure and abort. 
is 0.50 seconds. This time starts at CLV detection of abort confirmation signal and ends with Orion's LAS Abort Motor at $80 \%$ thrust. A diagram of the timeline of events for a CLV failure and abort is shown in Figure 31.

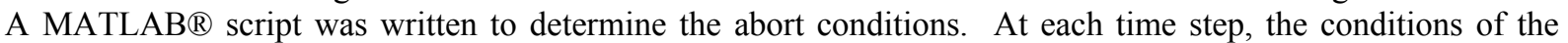
CLV as defined above are compared to the abort trigger conditions for the corresponding altitude or velocity; 0.50 seconds after one of the conditions is exceeded, the abort conditions are captured. In this way, abort conditions for every available CLV trajectory with a simulated failure are obtained.

The failure trajectories were split into three groups: 1) actuator lock in place and lock at null for the LF ISS Aug Open mission, 2) actuator lock in place and lock at null for the LF DE Feb Close mission, and 3) actuator lock at hard-over for the LF ISS Aug Open mission. Figure 32 is a comparison of the abort envelopes for these three cases. Angle-of-attack, sideslip angle, and the body rates are shown.

Failure types other than actuator failures are also being investigated for future analysis. One such example is a CLV case rupture where thrust is suddenly leaked from the side of the vehicle causing a disturbance torque.

LAS abort performance analysis is being conducted using the abort envelopes and abort conditions described here to assess the vehicle's ability to meet performance requirements.
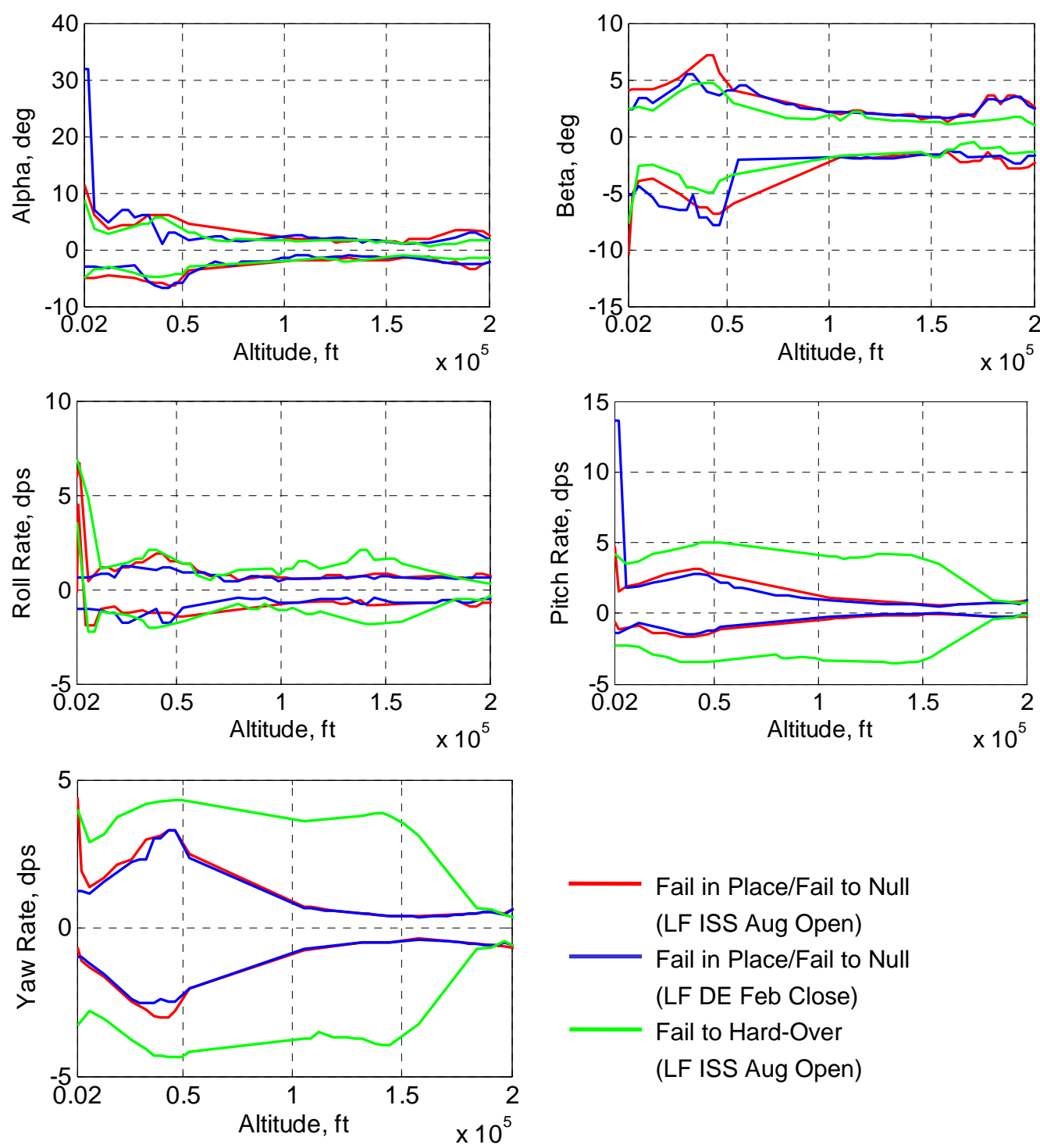

Figure 33. Comparison of abort envelopes due to failures. 


\section{Concluding Remarks}

This paper presented an overview of the Orion Crew Exploration Vehicle (CEV), an overview of the LAS ascent abort mode, and trade studies currently underway to assess abort performance and controllability. One of the primary design drivers for the CEV is to ensure crew safety. CEV requirements specify that abort capability should be continuously available from the launch pad until the mission destination is reached. Aborts during the critical ascent flight phase require the design and operation of CEV systems to escape from the Crew Launch Vehicle (CLV) and return the crew safely to the Earth. Several ascent abort modes have been designed and analyzed to accommodate the velocity, altitude, atmospheric, and vehicle configuration changes that occur during ascent. Aborts using the LAS, referred to as LAS aborts, provide abort coverage extending from the launch pad until early into the flight of the CLV second stage.

Simulation studies and design trades are being conducted so that more informed decisions can be made regarding the vehicle requirements, design, and operations. Examples of three analyses being used to assess LAS abort flight dynamic performance are: Launch Abort System Performance Analysis, Static Controllability Envelope Analysis, and Launch Abort Vehicle Tip-Off Conditions Due to Crew Launch Vehicle Failures and Dispersions. Summaries for those three analyses are described in the sections below.

The baseline CEV has been tested with the Government and Contractor simulation tools to evaluate LAS abort performance and capability to meet the current CEV abort performance requirements, from launch on a typical mission to the ISS.

The Launch Abort System Performance Analysis examined the performance and controllability of the current Launch Abort System abort configuration (LAV), which includes an extended aerodynamic faring that shields the $\mathrm{CM}$ from debris and the aero-thermal environment during ascent. The controllability analysis included a survey of non-dispersed aborts conducted at regular intervals along a nominal CLV ascent trajectory, as well as Monte Carlo dispersed aborts conducted from the launch pad, maximum dynamic pressure, and high altitude abort flight conditions. LAS abort performance metrics were discussed and then assessed for each abort scenario.

A static controllability tool has been developed to provide a way of visualizing the dynamics of different flight regimes and vehicle configurations and provides a way to investigate the reasons for some of the vehicle flight dynamics exhibited by the simulations. The tool has already proven valuable by providing an explanation for some of the behaviors of the LAS in the maximum dynamic pressure abort regime, as well as showing key differences in the performance of the flight test vehicle and operational vehicle configurations.

Over 40,000 CLV trajectories have been analyzed to obtain abort envelopes for the design and performance analysis of the LAV. The abort envelopes were defined in groups according to the type of failure simulated. An algorithm was developed to automatically create envelopes around the abort conditions for each type of failure. This algorithm was successful at allowing a quick definition of the abort envelopes. An important first look at the worst case abort conditions for the LAV was presented, allowing analysis of the vehicle's performance with these initial conditions.

\section{References}

1“"Exploration Systems Architecture Study Final Report,” NASA TM-2005-214062, November 2005.

2“Crew Exploration Vehicle System Requirements Document," NASA CEV Document Number: CxP-72000, January 2007.

${ }^{3}$ Davidson, J., et al., "Crew Exploration Vehicle Ascent Abort Overview," AIAA-2007-6590, AIAA Guidance, Navigation, \& Control Conference, Hilton Head, SC, August 2007.

4" Constellation Program Design Specification for Natural Environments (DSNE)," NASA Document Number: CxP-70023, January 2007.

5“Constellation Program Human-Systems Integration Requirements (HSIR)," NASA Document Number: CxP-70024, January 2007.

${ }^{6 “}$ Crew Exploration Vehicle Subsystem Requirements Specification Guidance, Navigation, \& Control,” NASA Document CEV-T-031210, July 2007.

7"“Crew Exploration Vehicle Architecture Design Document," NASA CEV Document Number: CxP-72140, January 2007.

8 Tedesco, M. et al., "Service Module Abort Preliminary Design," AIAA-2008-7150, AIAA Guidance, Navigation, \& Control Conference, August 2008.

${ }^{9}$ McCarthy, J. F. JR., Dodds, J. Ian, Crowders, R. S., "Development of the Apollo Launch Escape System,” J. Spacecraft, Vol. 5, No. 8, p. 927, North American Rockwell Corporation, Downey, CA, Aug. 1968.

${ }^{10}$ Sparks, D. and Raney, D., "Crew Exploration Vehicle Launch Abort System Controller Performance Analysis," AIAA2007-6595, AIAA Guidance, Navigation, and Control Conference, Hilton Head, SC, August 2007. 
${ }^{11}$ Acevedo, A. et al.,"ANTARES: Spacecraft Simulation for Multiple User Communities and Facilites." AIAA-2007-6888, AIAA Guidance, Navigation, \& Control Conference, Hilton Head, SC, August 2007.

${ }^{12}$ Justus, C. G., Johnson, D. L., "The NASA/MSFC Global Reference Atmospheric Model - 1999 Version (GRAM-99), NASA-TM-1999-209630, May 1999. 